\title{
Non-Abelian Fermionization and the Landscape of Quantum Hall Phases
}

\author{
Hart Goldman ${ }^{1, \psi}$, Ramanjit Sohal ${ }^{2, \chi}$, and Eduardo Fradkin ${ }^{2}$ \\ 1 Department of Physics, Massachusetts Institute of Technology, Cambridge, MA 02139, USA \\ 2 Department of Physics and Institute for Condensed Matter Theory, \\ University of Illinois at Urbana-Champaign, Urbana, Illinois 61801, USA
}

December 3, 2020

\begin{abstract}
The recent proposal of non-Abelian boson-fermion dualities in $2+1$ dimensions, which morally relate $U(k)_{N}$ to $S U(N)_{-k}$ Chern-Simons-matter theories, presents a new platform for exploring the landscape of non-Abelian quantum Hall states accessible from theories of Abelian composite particles. Here we focus on dualities relating theories of Abelian quantum Hall states of bosons or fermions to theories of non-Abelian "composite fermions" partially filling Landau levels. We show that these dualities predict special filling fractions where both Abelian and non-Abelian composite fermion theories appear capable of hosting distinct topologically ordered ground states, one Abelian and the other a non-Abelian, $U(k)_{2}$ Blok-Wen state. Rather than being in conflict with the duality, we argue that these results indicate unexpected dynamics in which the infrared and lowest Landau level limits fail to commute across the duality. In such a scenario, the non-Abelian topological order can be destabilized in favor of the Abelian ground state, suggesting the presence of a phase transition between the Abelian and non-Abelian states that is likely to be first order. We also generalize these constructions to other non-Abelian fermion-fermion dualities, in the process obtaining new derivations of a variety of paired composite fermion phases using duality, including the anti-Pfaffian state. Finally, we describe how, in multilayer constructions, excitonic pairing of the composite fermions across $N$ layers can also generate the family of Blok-Wen states with $U(k)_{2}$ topological order.
\end{abstract}

$\psi \leftrightarrow \chi$ These authors contributed equally to the development of this work. 


\section{Contents}

1 Introduction $\quad 1$

2 Review of non-Abelian dualities and the Landau-Ginzburg approach 5

2.1 Non-Abelian dualities and the $\nu=1 / 2$ bosonic Laughlin state . . . . . . . 5

2.2 A comment on level-rank duality and topological orders of fermions . . . . . 6

3 Non-Abelian dualities and the dynamics of composite fermions $\quad 7$

3.1 The $\nu=1 / 2$ Laughlin state and a non-Abelian fermion-fermion duality . . . 7

3.2 Abelian and non-Abelian Jain sequences . . . . . . . . . . . . . . . 9

3.3 Dynamical scenario . . . . . . . . . . . . . . . . . . . 11

3.4 Comments on the nature of the transition . . . . . . . . . . . . . . 14

3.5 Non-Abelian duality and paired FQH phases . . . . . . . . . . . . . . 15

3.6 Examples in other fermion-fermion dualities . . . . . . . . . . . . . . 17

4 Building non-Abelian states from excitonic pairing $\quad 20$

5 Discussion $\quad 23$

$\begin{array}{ll}\text { A Chern-Simons Conventions } & 24\end{array}$

B Details of $U(N)$ Fermion-Fermion Duality Examples 25

B.1 Derivation of the Duality . . . . . . . . . . . . . . 26

B.2 Examples involving gapless states . . . . . . . . . . . . . . . 27

B.3 Examples involving gapped states . . . . . . . . . . . . . . . 29 


\section{Introduction}

Two-dimensional electron fluids in strong magnetic fields are capable of hosting a rich tapestry of gapped, incompressible phases. The most famous among them occur at partial Landau level (LL) fillings $\nu$ and have fractionally quantized Hall conductivities $\sigma_{x y}=\nu \frac{e^{2}}{h}$, in what is known as the fractional quantum Hall $(\mathrm{FQH})$ effect. An explanation of the large family of FQH phases observed in experiments thus far, as well as an understanding of their underlying topological orders, was a major achievement of the past several decades. This progress is built on the unifying framework of flux attachment [1], which relates the original problem of electrons to a new problem of composite fermions or bosons coupled to a fluctuating Chern-Simons gauge field [2]. For example, in the language of flux attachment, the observed Abelian FQH states may be understood as arising from the formation of integer

quantum Hall (IQH) states of composite fermions [3, 4], or, equivalently, the condensation of composite bosons [5, 6].

Beyond the experimentally visible FQH states, an even more diverse array of quantum Hall phases have been proposed theoretically, many of which host quasiparticles with nonAbelian braiding statistics [7-11]. These proposals generally lack reference to microscopic physics, instead being based on "ideal" wave functions. Indeed, a general characterization of the dynamics that may lead to such non-Abelian phases has proven elusive, and it is unclear which of these phases are accessible from physically motivated theories of (Abelian) composite particles. With some notable exceptions, in which non-Abelian FQH states are obtained as paired states of composite fermions (the Moore-Read state) [12] or composite bosons (the Read-Rezayi sequences) [13-15], the composite particle picture has been largely unsuccessful in accessing the wider landscape of conjectured non-Abelian FQH states.

In recent work [15], we made progress on mapping the region of the non-Abelian FQH landscape accessible to theories of Abelian composite particles. We developed LandauGinzburg theories for a large class of non-Abelian states which are related to Abelian composite particle theories via recently proposed Chern-Simons-matter theory dualities [16]. Motivated by the equivalence of $U(N)_{k}$ Chern-Simons theories coupled to gapless complex bosons and $S U(k)_{-N}$ theories coupled to gapless Dirac fermions in the 't Hooft (large- $N, k$ ) limit $[17,18]$, these dualities relate theories of gapless bosons or fermions coupled to ChernSimons gauge theories in a manner which parallels the established level-rank dualities of pure Chern-Simons theories [19-22]. Of these dualities, several relate theories with Abelian and non-Abelian gauge groups, meaning that they represent dualities between the conventional composite boson Landau-Ginzburg theories for certain Abelian FQH states and theories of 
dual bosons coupled to non-Abelian Chern-Simons gauge fields. ${ }^{1}$ By stacking multiple copies of these Abelian states and introducing pairing in the dual non-Abelian composite boson language, we showed that one can access the Read-Rezayi [10] and generalized non-Abelian spin singlet states [23-25]. The success of this construction stems from the observation that phases naturally accessible by condensing local operators in the non-Abelian dual theory may not be visible in the original Abelian theory, in which these operators correspond to non-local monopole operators.

Lying at the heart of our construction is a string of dualities involving the usual LandauGinzburg theory for the $\nu=1 / 2$ bosonic Laughlin state, a single flavor of Wilson-Fisher boson (describing the Laughlin quasiparticles) coupled to a $U(1)_{2}$ Chern-Simons gauge field. This theory has three duals,

a Wilson-Fisher scalar $+U(1)_{2} \longleftrightarrow$ a Dirac fermion $+S U(2)_{-1 / 2}$

a Dirac fermion $+U(1)_{-3 / 2} \longleftrightarrow$ a Wilson-Fisher scalar $+S U(2)_{1}$,

where we use $\longleftrightarrow$ to denote duality and subscripts denote the Chern-Simons level (including the parity anomaly). The Abelian boson-fermion duality featured here and others like it were explored in Refs. [26-29]. While most non-Abelian dualities are boson-fermion dualities, this quadrality - in which each of the four theories is dual to the others - is distinguished by its inclusion of non-Abelian boson-boson and fermion-fermion dualities. In Ref. [15], we focused on the non-Abelian boson-boson duality, in which the dual theory consists of non-Abelian bosonic "composite vortices" coupled to a $S U(2)_{1}$ Chern-Simons gauge field, obtaining the non-Abelian phases via inter-layer pairing of the composite vortices.

In this work, we study the non-Abelian phases accessible to the dual theories of composite fermions. Of these, the theory of Dirac fermions coupled to a $U(1)_{-3 / 2}$ gauge field is a relativistic version of the standard composite fermion description of the $\nu=1 / 2$ bosonic Laughlin state, while the theory of Dirac fermions coupled to a $S U(2)_{-1 / 2}$ gauge field constitutes a different kind of "flux attachment" in which the composite fermions possess charge under a fluctuating non-Abelian gauge field. Using this duality, we analyze two of the simplest paths to non-Abelian phases:

\footnotetext{
${ }^{1}$ We emphasize here that it is possible for a Chern-Simons gauge theory to have a non-Abelian gauge group but an Abelian braid group, meaning that it represents an Abelian topological phase. For example, $S U(2)_{1}$ is Abelian in this sense, having the same anyon content as $U(1)_{2}$ by level-rank duality.
} 
1. Forming integer quantum Hall (IQH) phases of the $S U(2)$ composite fermions, in analogy with Jain's construction of Abelian FQH phases as IQH states of composite fermions [3] and earlier projective parton constructions of non-Abelian FQH states $[7,30,31]$.

2. Excitonic pairing between layers of $S U(2)$ composite fermions. This construction is a composite fermion version of the one presented in Ref. [15] for composite bosons.

In both constructions, we will find that the composite fermions yield the $\nu=k / 2$ Blok-Wen states with $U(k)_{2}$ topological order [9], in contrast to the Read-Rezayi states obtained via inter-layer pairing of the $S U(2)$ bosons in Ref. [15]. For many of these states, these are the first constructions starting from parent theories of Abelian composite particles, rather than projective parton constructions [30-32] or more general non-Abelian/non-Abelian dualities [33]. In addition, by considering more general non-Abelian dualities, we find not only the exotic Fibonacci state [34], but also composite fermion descriptions of a variety of nonAbelian states that have previously been understood via pairing instabilities of a composite Fermi liquid, including a new description of the anti-Pfaffian state [35, 36]. Remarkably, we find in these special cases that an IQH phase of the non-Abelian composite fermion theory is dual to pairing in the usual Abelian description. Our construction of all of these states using non-Abelian dualities represents the first category of main results of this work.

In addition to revealing paths to different non-Abelian phases, these non-Abelian fermionfermion dualities possess surprising information about the dynamics of composite fermions, leading to our second main family of results. These results relate to our first construction of the $U(k)_{2}$ states, in which a magnetic field and chemical potential are adjusted so that the $S U(2)$ composite fermions fill $k$ Landau levels. This leads to a $S U(2)_{-k}$ spin topological quantum field theory (spin TQFT) at low energies, corresponding to $U(k)_{2}$ topological order by level-rank duality. However, this conclusion is immediately complicated by the duality with the Abelian composite fermion theory, IQH phases of which correspond to the bosonic Jain sequence states. Indeed, there are certain filling fractions $\nu_{*}$ of the underlying electric charges at which both types of composite fermions fill up an integer number of Landau levels. On integrating out the fermions in these two theories, one would then be led to conclude that the theory with an Abelian gauge group predicts an Abelian FQH state, while the nonAbelian theory predicts a non-Abelian FQH state. For instance, for a system of bosons at filling $\nu_{*}=3 / 2$, the two theories appear to respectively predict $U(1)_{-2}$ and $U(3)_{2}$ topological order.

Because $U(3)_{2}$ and $U(1)_{-2}$ are distinct topological orders and are certainly not dual to 


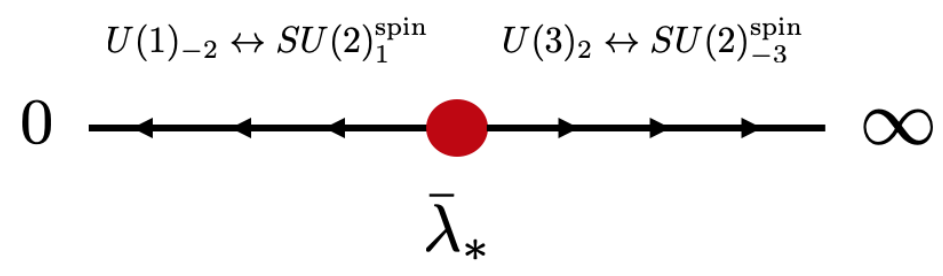

Figure 1: Proposed phase diagram for the $S U(2)$ composite fermion theory at filling $\nu=3 / 2$. Here $\bar{\lambda}=g_{Y M}^{2} / \omega_{c}$, where $g_{Y M}^{2}$ is the Yang-Mills coupling (the fine structure constant) and $\omega_{c} \sim \sqrt{B}$ is the cyclotron frequency. When $\bar{\lambda} \rightarrow \infty$, the Yang-Mills term vanishes, and the picture of deconfined composite fermions filling (color degenerate) Landau levels is valid, leading to the $S U(2)_{-3}^{\text {spin }} \leftrightarrow U(3)_{2}$ state. When $\bar{\lambda}$ runs small, the Yang-Mills term becomes very large, Landau levels can mix, and the deconfinement of the composite fermions is no longer assured, leading ultimately to the $U(1)_{2}$ phase predicted in the dual Abelian theory. These states are separated by a critical point at $\bar{\lambda}=\bar{\lambda}_{*} \sim \mathcal{O}(1)$, which is likely first order.

one another, one might naïvely worry that these results signal a breakdown of the dualities, which postulate an equivalence of the infrared (IR) limits, or ground states, of the dual theories. On the other hand, it is very common for states with distinct topological orders to exist at the same filling fraction, with the ultimate choice of ground state depending on details of local energetics. Indeed, in this work we take the view that both topological orders are valid ground states at filling $\nu_{*}$, and that the particular choice of ground state depends on the order in which the lowest Landau level $(B \rightarrow \infty)$ and IR limits are taken. This order of limits is subtle, as the duality is only valid in the IR limit, while the statement that the composite fermions form a stable IQH state relies on the $B \rightarrow \infty$ limit. More precisely, on tuning the ratio of the Yang-Mills coupling to the cyclotron frequency in the non-Abelian theory, we argue that there is a phase transition between the Abelian and non-Abelian FQH states (see Figure 1). Such a transition, if continuous, would be quite exotic, as it would separate two very different topological orders and therefore lie beyond the Landau-Ginzburg paradigm. Thus, when the $U(1)$ composite fermions form an IQH state, the $S U(2)$ composite fermions experience an instability and find themselves on the Abelian side of this transition and vice versa.

Even if this transition is first order, our results demonstrate that QFT dualities can be used to infer non-trivial statements about the phase diagrams and dynamics of ChernSimons-matter theories in the presence of background fields. Indeed, the phenomenon described above is a general feature of fermion-fermion dualities involving non-Abelian gauge groups, and we collect several additional examples. These appear both in the $S U(2)$ quadrality discussed above as well as in other dualities. In particular, we consider the case of the 
duality between a free Dirac fermion and a Dirac fermion coupled to a $U(N)_{-1 / 2}$ gauge field. We also comment that the scenario we present is reminiscent of recent proposals for the phase diagram of $S U(N)_{k}$ Chern-Simons theory with $N_{f}>2 k$ fermion flavors (at zero density and magnetic field) [37] and recent follow-up work [38-41], in which it has been suggested that the Yang-Mills term can play a non-trivial role despite superficially appearing irrelevant (in the sense of the renormalization group).

This work is organized as follows. In Section 2, we review the non-Abelian Chern-Simonsmatter dualities, focusing on the quadrality in Eq. (1.1), and we review the construction of Ref. [15]. In Section 3, we present a detailed description of the dual fermionic theories and the properties of their IQH states, and we present the cases in which they appear to predict different topological orders. We then present our proposed scenario for how this state of affairs can be made consistent and provide additional examples of dual fermionic theories displaying similar phenomena. In the process, we uncover an entire series of nonAbelian FQH states, including the anti-Pfaffian [35, 36], which we find can be simultaneously described as arising from IQH states of composite fermions in a non-Abelian theory and from pairing instabilities of a composite Fermi liquid in a dual Abelian theory. In Section 4, we demonstrate how the $U(k)_{2}$ states can be obtained through stacking and excitonic pairing in Abelian FQH states. Finally, we conclude with a discussion of our results and their implications.

\section{Review of non-Abelian dualities and the Landau-Ginzburg ap- proach}

\subsection{Non-Abelian dualities and the $\nu=1 / 2$ bosonic Laughlin state}

In this section, we first briefly review the dualities relevant to describing the $\nu=1 / 2$ bosonic Laughlin state. The recently proposed non-Abelian Chern-Simons-matter dualities relate theories of Wilson-Fisher bosons coupled to a Chern-Simons gauge field to theories of Dirac fermions also coupled to a Chern-Simons gauge field, with the matter content in the fundamental representation of the gauge group. We can write these dualities schematically as

$$
\begin{aligned}
& N_{f} \text { scalars }+U(N)_{k, k} \longleftrightarrow N_{f} \text { fermions }+S U(k)_{-N+N_{f} / 2}, \\
& N_{f} \text { scalars }+S U(N)_{k} \longleftrightarrow N_{f} \text { fermions }+U(k)_{-N+N_{f} / 2,-N+N_{f} / 2}, \\
& N_{f} \text { scalars }+U(N)_{k, k+N} \longleftrightarrow N_{f} \text { fermions }+U(k)_{-N+N_{f} / 2,-N-k+N_{f} / 2} .
\end{aligned}
$$


Our conventions and notation concerning non-Abelian Chern-Simons theories are presented in Appendix A.

The dualities, Eqs. (2.1)-(2.3), can be shown to imply the quadrality described in the Introduction [22],

$$
\begin{gathered}
\text { a scalar }+U(1)_{2} \longleftrightarrow \text { a fermion }+S U(2)_{-1 / 2} \\
\uparrow \\
\text { a fermion }+U(1)_{-3 / 2} \longleftrightarrow \text { a scalar }+S U(2)_{1} .
\end{gathered}
$$

The first theory (top left) is the relativistic version of the usual Landau-Ginzburg theory for the $\nu=1 / 2$ bosonic Laughlin state. Explicitly, it is described by the Lagrangian,

$$
\mathcal{L}_{\Phi}=\left|D_{a} \Phi\right|^{2}-|\Phi|^{4}+\frac{2}{4 \pi} a d a+\frac{1}{2 \pi} A d a .
$$

Here $a$ is an emergent $U(1)$ gauge field; $A$ the background electromagnetic (EM) field; we use the notation $D_{a}^{\mu}=\partial^{\mu}-i a^{\mu}$ and $a d b=\varepsilon^{\mu \nu \lambda} a_{\mu} \partial_{\nu} b_{\lambda}$; and the term $-|\Phi|^{4}$ denotes tuning to the Wilson-Fisher fixed point. The dual non-Abelian bosonic theory (bottom right) is given by

$$
\mathcal{L}_{\phi}=\left|D_{u-A \mathbf{1} / 2} \phi\right|^{2}-|\phi|^{4}+\frac{1}{4 \pi} \operatorname{Tr}\left[u d u-\frac{2 i}{3} u^{3}\right]-\frac{1}{2} \frac{1}{4 \pi} A d A,
$$

where $u$ is an $S U(2)$ gauge field, 1 is the $2 \times 2$ identity matrix in color space, and $-|\phi|^{4}$ again denotes tuning to the Wilson-Fisher fixed point. By turning on mass operators, both theories can be shown to describe the transition between the $\nu=1 / 2$ bosonic Laughlin state and the trivial insulator, which correspond to their gapped $(\langle\Phi\rangle=0,\langle\phi\rangle=0)$ and condensed $(\langle\Phi\rangle \neq 0,\langle\phi\rangle \neq 0)$ phases, respectively. Essential to this conclusion is the fact that $S U(2)_{1}$, the TQFT obtained when $\Phi$ is gapped, is Abelian at the level of the braid group: it is equivalent to $U(1)_{2}$ by level-rank duality.

The $S U(2)$ theory, $\mathcal{L}_{\phi}$, served as the main building block in our construction of the Read-Rezayi states in Ref. [15], in which we considered multiple layers of the $\nu=1 / 2$ bosonic Laughlin state and introduced an interlayer pairing interaction for the $\Phi$ particles. The paired phase of this theory yielded the Read-Rezayi states. However, in that work we did not consider the landscape of non-Abelian phases accessible by dual theories of Dirac fermions, which we now turn to.

\subsection{A comment on level-rank duality and topological orders of fermions}

Before describing the composite fermion theories of interest, we mention here a subtlety that arises when considering topological orders of composite fermions. When assessing the 
anyon content of the corresponding gauge theory, it is necessary to account for the the fact that the degrees of freedom charged under the gauge field are fermions, which affects the statistics of certain anyons by a minus sign. More technically, this is a result of the fact that gauge fields which couple to fermions are spin (actually $\operatorname{spin}_{c}$, see Appendix A) connections, as opposed to the $U(1)$ connections that couple to bosons. We will refer to such gauge fields throughout this paper as spin gauge fields, and we will denote their associated TQFTs with the superscript 'spin.' In general, level-rank duality can be thought of as relating a TQFT with a spin gauge field (spin TQFT) to one with a $U(1)$ gauge field. ${ }^{2}$ Therefore, since composite fermion theories give rise to spin TQFTs, we will frequently invoke levelrank duality below and refer to a state's topological order via its corresponding (non-spin) TQFT. For example, if a composite fermion theory yields a $S U(2)_{-k}^{\text {spin }}$ TQFT, we will refer to the associated topological phase by its level-rank dual, $U(k)_{2}$.

This formal discussion has physical implications. For example, consider the topological order of the $\nu=1 / 2$ Laughlin state. The anyons of this state are semions, with $\pi / 2$ statistics. This state can be equally well described by a $U(1)_{2}$ TQFT or a $U(1)_{-2}$ TQFT with a spin gauge field, which we will denote $U(1)_{-2}^{\text {spin }}$. As we will see below, this theory arises on integrating out a Landau level of composite fermions. While it appears that the anyons in this theory are antisemions (statistics $-\pi / 2$ ), the $\pi$ statistics of the composite fermions converts them into semions. This is a type of level-rank duality, relating $U(1)_{2}$ to $U(1)_{-2}^{\text {spin }}$. In this sense, level-rank dualities can generally be viewed as boson-fermion dualities, with some interesting exceptions, e.g. in the $S U(2)_{1} \leftrightarrow U(1)_{2}$ duality mentioned above, neither theory is spin.

\section{Non-Abelian dualities and the dynamics of composite fermions}

\subsection{The $\nu=1 / 2$ Laughlin state and a non-Abelian fermion-fermion duality}

The bosonic theories described above are dual to a theory of Dirac fermions coupled to a $U(1)_{-3 / 2}$ Chern-Simons gauge field. For clarity, we will refer to this theory as Theory A,

$$
\mathcal{L}_{A}=i \bar{\psi} \not D_{a} \psi-\frac{3}{2} \frac{1}{4 \pi} a d a-\frac{1}{2 \pi} a d A-\frac{1}{4 \pi} A d A+\cdots
$$

\footnotetext{
${ }^{2}$ Level-rank duality can be equivalently formulated to relate two spin-TQFTs by adding an invisible spin$1 / 2$ line (also known in the condensed matter literature as a local spin- $1 / 2$ particle) to each side of the duality [22]. This formulation is less physical if we wish to view the fundamental charges at short distances as bosons, so we will refrain from using it.
} 
where $a$ is a $U(1)$ gauge field and we use the notation $\not D=D_{\mu} \gamma^{\mu}$, where $\gamma^{\mu}$ are the Dirac gamma matrices. This theory is also dual to a theory of Dirac fermions coupled to a nonAbelian, $S U(2)_{-1 / 2}$ gauge field, which we will refer to as Theory B,

$$
\mathcal{L}_{B}=i \bar{\chi} \not D_{b-A \mathbf{1} / 2} \chi-\frac{1}{2} \frac{1}{4 \pi} \operatorname{Tr}\left[b d b-\frac{2 i}{3} b^{3}\right]-\frac{1}{4} \frac{1}{4 \pi} A d A+\cdots
$$

where $b$ is an $S U(2)$ gauge field and $\mathbf{1}$ is the $2 \times 2$ identity matrix. ${ }^{3}$ Here the $\chi$ fields transform as a doublet under $S U(2)$, and they have charge $-1 / 2$ under the global EM symmetry, $U(1)_{\mathrm{EM}}$. The fundamental (unit) charges are therefore the baryons, $\varepsilon_{\alpha \beta} \chi^{\alpha} \chi^{\beta}$, where $\alpha, \beta=1,2$ are $S U(2)$ color indices. Finally, the ellipses refer to irrelevant operators, such as Maxwell or Yang-Mills terms for the gauge fields. These operators are normally dropped since the duality is only valid in the IR limit, in which these operators are taken to zero, and their usual purpose is to provide UV regularization. However, we will see in the sections below that these operators can play important roles in determining low energy physics when background fields are turned on.

Being dual to the bosonic theories discussed in Section 2, Theory A and Theory B each describe a transition from the $\nu=1 / 2$ bosonic Laughlin state, which has $U(1)_{2}$ topological order, to a trivial insulator. This can be seen by introducing mass terms, $-m_{\psi} \bar{\psi} \psi$ and $-m_{\chi} \bar{\chi} \chi$, to their respective theories. For $m_{\psi}>0, m_{\chi}>0$, integrating out $\psi$ and $\chi$ can be seen to immediately yield an insulating state with vanishing Hall conductivity, $\sigma_{x y}=0$. On the other hand, when $m_{\psi}<0, m_{\chi}<0$, integrating out the composite fermions yields a state with $\sigma_{x y}=-\frac{1}{2} \frac{1}{2 \pi}\left(m_{\psi}<0, m_{\chi}<0\right)$, with Theory A yielding a $U(1)_{-2}^{\text {spin }}$ gauge theory and Theory B yielding $S U(2)_{-1}^{\text {spin }}$. These constitute the same topological order as the $U(1)_{2}$ state by level-rank duality.

By differentiating this pair of Lagriangians with respect to the background EM gauge field, $A_{\mu}$, to obtain the global EM charge current, $J^{\mu}$, one observes that, under the duality, the monopole current of Theory $\mathbf{A}$ is related to the baryon number current of Theory B,

$$
J_{e}^{\mu}=\frac{1}{2 \pi} \varepsilon^{\mu \nu \lambda} \partial_{\nu}\left(a_{\lambda}-A_{\lambda}\right) \leftrightarrow-\frac{1}{2} j_{\chi}^{\mu}-\frac{1}{2} \frac{1}{2 \pi} \varepsilon^{\mu \nu \lambda} \partial_{\nu} A_{\lambda}
$$

where $j_{\chi}^{\mu}=\bar{\chi} \gamma^{\mu} \chi$ is the $\chi$ charge current of the Theory B. The interpretation of this dictionary is analogous to charge-vortex duality [42,43]: flux of the gauge field $a$ in Theory A maps to charge of the $\chi$ fermions in Theory B. The same interpretation applies to the pair of bosonic theories discussed in the previous Section.

\footnotetext{
${ }^{3}$ Throughout this work, we approximate the Atiyah-Patodi-Singer $\eta$-invariant as a level- $\frac{1}{2}$ Chern-Simons term and explicitly include it in the Lagrangian.
} 


\subsection{Abelian and non-Abelian Jain sequences}

In contrast to their bosonic counterparts, these composite fermions each satisfy the Pauli exclusion principle. As a result, it is natural to consider the gapped phases accessible by filling up Landau levels and forming IQH states, in analogy to the construction of the Jain sequences, in which FQH phases are obtained as IQH states of composite fermions [3]. In particular, integer quantum Hall states of the $S U(2)$ doublet composite fermions, $\chi$, can be expected to yield non-Abelian topological orders. This method of forming non-Abelian quantum Hall phases appears to be quite natural, but we will quickly learn that the nonAbelian dualities imply that things are not so simple, and the ultimate choice of ground state will be sensitive to the order in which the lowest Landau level and IR limits are taken.

To this end, we begin by relating the electronic filling fraction, $\nu$, to the filling fractions of the $\psi$ and $\chi$ fermions using the dictionary, Eq. (3.3). Focusing first on the Abelian Theory A, the physical electric charge density is given in terms of the magnetic flux felt by the composite fermions,

$$
\rho_{e}=\left\langle J_{e}^{0}\right\rangle=-\frac{1}{2 \pi}\left\langle\varepsilon^{i j} \partial_{i} a_{j}\right\rangle-\frac{1}{2 \pi} B
$$

where $B=\varepsilon^{i j} \partial_{i} A_{j}$ is the background magnetic field. We use brackets here to emphasize that that we define $\rho_{e}$ to be the expectation value of the charge density operator. We can relate $\rho_{e}$ to the composite fermion charge density through the equation of motion for $a_{0}$,

$$
0=\left\langle\psi^{\dagger} \psi\right\rangle-\frac{3}{4 \pi}\left\langle\varepsilon^{i j} \partial_{i} a_{j}\right\rangle-\frac{1}{2 \pi} B
$$

If we define the composite fermion filling fraction of Theory $\mathbf{A}$ to be

$$
\nu_{\psi}=2 \pi \frac{\left\langle\psi^{\dagger} \psi\right\rangle}{\left\langle\varepsilon^{i j} \partial_{i} a_{j}\right\rangle},
$$

we obtain a relation between the composite fermion and electronic filling fractions,

$$
\nu=-2 \pi \frac{\rho_{e}}{B}=\frac{\nu_{\psi}-1 / 2}{\nu_{\psi}-3 / 2} .
$$

Note that we have absorbed a minus sign into the definition of $\nu$ for notational convenience. From this formula, we see that IQH states of the $\psi$ fermions, which occur at fillings $\nu_{\psi}=$ $p-1 / 2$, correspond to the known (descendent) bosonic Jain sequence states,

$$
\nu_{p}=\frac{p-1}{p-2}, p \in \mathbb{Z} .
$$


Indeed, integrating out the composite fermions yields the Lagrangian,

$$
\mathcal{L}_{A, \mathrm{eff}}=\frac{p-2}{4 \pi} a d a-\frac{1}{2 \pi} a d A-\frac{1}{4 \pi} A d A .
$$

Each of these states (with the exception of the states at $p=1,2$, which are respectively a trivial insulator and a superfluid) is an Abelian FQH phase of the physical charges, which here are bosons.

The same type of analysis can be carried out for Theory $\mathbf{B}$, leading to a non-Abelian version of the bosonic Jain sequence. Recalling Eq. (3.3), the electric charge density is directly related to the density of $\chi$ fermions, $\chi^{\dagger} \chi$, via

$$
\rho_{e}=-\frac{1}{2}\left\langle\chi^{\dagger} \chi\right\rangle-\frac{1}{4} \frac{1}{2 \pi} B
$$

Because the $\chi$ fermions are coupled to a Chern-Simons gauge field, they do not confine, meaning that a non-zero magnetic field, $B$, will cause them to form Landau levels with degeneracy,

$$
d_{L L}=\frac{B A}{2 \pi} \times\left|q_{\chi}\right| \times(\text { color degeneracy })=\frac{B A}{2 \pi},
$$

where $A$ in this expression is the area of the system and $q_{\chi}=-1 / 2$ is the EM charge of the $\chi$ fermions. Therefore, the filling fraction of the $\chi$ fermions is

$$
\nu_{\chi}=2 \pi \frac{\left\langle\chi^{\dagger} \chi\right\rangle}{B}
$$

Plugging this into Eq. (3.10) yields a relation between $\nu$ and $\nu_{\chi}$,

$$
\nu=\frac{1}{2} \nu_{\chi}+\frac{1}{4}
$$

When the $\chi$ fermions fill an integer number of Landau levels, $\nu_{\chi}=s-1 / 2$, and the filling of the physical charges is

$$
\nu_{s}=\frac{s}{2}, s \in \mathbb{Z}
$$

On integrating out the composite fermions, one obtains a $S U(2)_{-s}^{\text {spin }}$ theory with Lagrangian,

$$
\mathcal{L}_{B, \text { eff }}=-\frac{s}{4 \pi} \operatorname{Tr}\left[b d b-\frac{2 i}{3} b^{3}\right]-\frac{s}{2} \frac{1}{4 \pi} A d A,
$$

which describes a non-Abelian, $U(s)_{2}$, topological order when $|s|>1$. This approach recalls earlier approaches to non-Abelian FQH states using parton constructions [7, 30, 31]. However, unlike in those cases, in which the elctron operator is fractionalized by hand and it is 
necessary to require that the partons do not confine by fiat, here the duality provides a clear basis for the presence of a (deconfining) non-Abelian gauge field.

Having obtained the sequences of incompressible filling fractions associated with Theory A and Theory B, one can immediately observe several special (non-trivial) filling fractions, $\nu_{*}$, at which they coincide, indicating the presence of competing ground states. Comparing Eqs. (3.8) and (3.14), these fillings occur when $\nu_{p}=\nu_{s}=\nu_{*}$, i.e.

$$
s=2\left(1+\frac{1}{p-2}\right), p, s \in \mathbb{Z} .
$$

Here we recall that $p$ and $s$ are the number of Landau levels filled by the $\psi$ and $\chi$ fermions, respectively. This equation has several solutions, which are organized in Table 1. Two of these solutions, $(p=1, s=0)$ and $(p=0, s=1)$, respectively correspond to the $\nu=1 / 2$ bosonic Laughlin state, which has $U(1)_{2} \leftrightarrow S U(2)_{-1}^{\text {spin }}$ topological order. This is consistent with the fact that at criticality these theories describe a plateau transition between these states.

It is the presence of other solutions, at $(p=3, s=4)$ and $(p=4, s=3)$, corresponding to $\nu_{*}=2$ and $\nu_{*}=3 / 2$ respectively, that reveals new physics. On the one hand, Theory A predicts the $\nu_{*}=3 / 2$ and $\nu_{*}=2$ states to have $U(1)_{-2}$ and $U(1)_{1}$ (i.e. trivial) topological orders. On the other hand, Theory $\mathbf{B}$ predicts the same states to have non-Abelian $S U(2)_{-3}^{\text {spin }} \leftrightarrow U(3)_{2}$ and $S U(2)_{-4}^{\text {spin }} \leftrightarrow U(4)_{2}$ topological orders. While it is common in quantum Hall physics for different competing states to be proposed for the same filling fraction, the duality of Theory A and Theory B identifies the two theories' ground states. Therefore, the consistency of the duality implies that the conditions under which the $\psi$ fermions form an IQH state are not the same as those of the $\chi$ fermions, and the two possible states must be separated by a phase transition. A theory of this phase transition requires shortdistance dynamical information not specified by, but consistent with, the duality. We now present a possible scenario for a transition of this kind.

\subsection{Dynamical scenario}

We now provide a possible explanation of the physics occurring at the special filling fractions $\nu_{*}=3 / 2,2$. For now, we will work from the point of view of the non-Abelian Theory B, and we will begin by considering what happens as we fill Landau Levels. From Table 1, we see that filling the zeroth and first Landau Levels of the $\chi$ fermions corresponds to the expected trivial insulator $(\nu=0)$ and bosonic Laughlin $(\nu=1 / 2)$ states. What occurs when the non-Abelian composite fermions fill two Landau levels is also quite non-trivial, but we will 


\begin{tabular}{|c||c|c|c|c|}
\hline$\nu_{*}$ & 0 & $\frac{1}{2}$ & $\frac{3}{2}$ & 2 \\
\hline$\nu_{\psi}+1 / 2$ & 1 & 0 & 4 & 3 \\
\hline$\nu_{\chi}+1 / 2$ & 0 & 1 & 3 & 4 \\
\hline Theory A & Trivial & $U(1)_{2}$ & $U(1)_{-2}$ & IQH \\
\hline Theory B & Trivial & $U(1)_{2}$ & $U(3)_{2}$ & $U(4)_{2}$ \\
\hline
\end{tabular}

Table 1: Solutions to Eq. (3.16), in which both the $\psi$ and $\chi$ composite fermions form IQH states at special electronic filling fractions, $\nu_{*}$. Also indicated are the topological orders predicted by the dual theories for each filling.

table that discussion until the next subsection. For now, our concern will be what happens when we fill the third Landau level, corresponding to $\nu_{*}=3 / 2$. Our proposal for $\nu_{*}=2$ will prove to be essentially identical. At $\nu_{*}=3 / 2$, Theory $\mathbf{B}$ predicts an incompressible state with $S U(2)_{-3}^{\text {spin }} \leftrightarrow U(3)_{2}$ topological order, while Theory A predicts $U(1)_{2}$. This suggests that it should be possible to trigger an instability in the non-Abelian theory as we fill this Landau level, which preempts the $U(3)_{2}$ topological order and yields the same Abelian phase predicted by Theory A (and vice versa). Consequently, both the $U(1)_{2}$ and $U(3)_{2}$ phases must exist in the $\nu=3 / 2$ phase diagram. This is the only state of affairs consistent with the duality.

How might such an instability occur? It is here that the ellipses in Eqs. (3.1) and (3.2) become crucial. ${ }^{4}$ These ellipses include operators that are irrelevant at tree level but may nevertheless play an important role in determining the low energy physics when a magnetic field and chemical potential are introduced. Indeed, there is no sense in which such fields are ever perturbative, as they reorganize the spectrum of a theory in dramatic ways. To make this discussion more precise, consider the Yang-Mills term in Theory B,

$$
\mathcal{L}_{Y M}=-\frac{1}{2 g_{Y M}^{2}} \operatorname{Tr}\left[f_{\mu \nu} f^{\mu \nu}\right],
$$

where $f_{\mu \nu}=\partial_{\mu} b_{\nu}-\partial_{\nu} b_{\mu}-i\left[b_{\mu}, b_{\nu}\right]$ is the field strength of the $S U(2)$ gauge field, $b$. At tree level, the mass dimension of the operator $\operatorname{Tr}\left[f^{2}\right]$ is 4 , meaning that $\left[g_{Y M}^{2}\right]=1$ : it is an energy scale. Commonly, the IR limit in which the duality of Eqs. (3.1) and (3.2) holds is phrased as the limit $g_{Y M}^{2} \sim \Lambda \rightarrow \infty$, where $\Lambda$ is a UV cutoff, but strictly speaking this is only true in the absence of a background magnetic field, which provides its own energy scale in the form of the cyclotron frequency, $\omega_{c} \sim \sqrt{B}$ (for massless Dirac fermions).

\footnotetext{
${ }^{4}$ We thank Chong Wang for enlightening discussions on this point.
} 
Consequently, one can form a dimensionless coupling,

$$
\bar{\lambda}=\frac{g_{Y M}^{2}}{\omega_{c}},
$$

that can have non-trivial running as a result of strong interaction effects. This would mean that the ground state ultimately chosen by Theory A can depend on the order of the limits, $g_{Y M}^{2} \rightarrow \infty$ and $\omega_{c} \rightarrow \infty$. Importantly, one order of limits in Theory B may not correspond to an analogous order of limits in Theory A. In other words, Theory $\mathbf{B}$ may be in a strongly coupled regime $(0 \leq \bar{\lambda}<\infty)$ while Theory A may behave as if all of the irrelevant operators have been taken to zero prior to taking $\omega_{c} \rightarrow \infty$.

This is the essence of our proposal ${ }^{5}$ for the phase diagram of Theory $\mathbf{B}$, shown schematically in Fig. 1. Whether the theory is in the $U(3)_{2}$ or $U(1)_{2}$ phase is determined by the value of $\bar{\lambda}$, with the two phases being separated by a phase transition at a value $\bar{\lambda}=\bar{\lambda}_{*} \sim \mathcal{O}(1)$. For $\bar{\lambda}>\bar{\lambda}_{*}, \bar{\lambda}$ runs large, corresponding to the limit $g_{Y M}^{2} \rightarrow \infty$ followed by $\omega_{c} \rightarrow \infty$. In this phase, the Yang-Mills term disappears, leaving the Chern-Simons term, which ensures that the composite fermions, $\chi$, are deconfined. It is in this regime that we expect the picture described in the previous subsection of deconfined $\chi$ fermions filling Landau levels to hold, making this the phase with $U(3)_{2}$ topological order. On the other hand, for $\bar{\lambda}<\bar{\lambda}_{*}$, we propose that $\bar{\lambda}$ runs small. Here the Yang-Mills term becomes important, and the assumption of deconfined composite fermions breaks down: the Landau levels mix. As a result, the composite fermions will tend to form bound states that are neutral under $S U(2)$ : the baryons, $\varepsilon_{\alpha \beta} \chi^{\alpha} \chi^{\beta}$. These are bosons of charge -1 since the fermions are doublets under $S U(2)$. Being the physical electric charges in this theory, these bosonic baryons will find themselves at filling $\nu=3 / 2$, and the resulting topological order will ultimately be $U(1)_{2}$, as predicted by Theory A. Note that this final conclusion is conjecture based on the consistency of the duality - the physics in such a phase is very strongly coupled, and we cannot show explicitly that this is the true ground state. We also point out that $\bar{\lambda}$ may not run to zero in this phase, instead running to a small but finite value. This represents a very interesting but theoretically daunting possibility.

We now comment on what occurs in the dual description of Theory A, in which we

\footnotetext{
${ }^{5}$ We emphasize that while we focus on the example of the Yang-Mills term, there are many other operators that might be responsible for the behavior we propose, and it may be more correct to consider a linear combination of these operators as being responsible. Another likely family of examples is four-fermion operators, which have the same tree level dimension as the Yang-Mills operator.
} 
can define a similar running coupling ${ }^{6}, \bar{\lambda}_{A}=g_{\text {Maxwell }}^{2} / \omega_{c}$, where $g_{\text {Maxwell }}$ is the coupling associated with the Maxwell term for the $U(1)$ gauge field, $a$. In this theory, the limit $\bar{\lambda}_{A} \rightarrow \infty$ corresponds to the $U(1)_{2}(\mathrm{IQH})$ state at filling $\nu_{*}=3 / 2\left(\nu_{*}=2\right)$, as the Maxwell term vanishes. However, since the Maxwell term is not dual to the Yang-Mills term, it is not clear whether the phase transitions described for Theory B correspond to transitions tuned by $\bar{\lambda}_{A}$ or another coupling, such as one associated with a linear combination of four-fermion operators. Nevertheless, unless $\bar{\lambda}_{*}=\infty$ in Theory B (in which case the non-Abelian phase is nowhere stable, having no basin of attraction), the duality indicates that the non-Abelian states should be accessible to Theory A as well, and that these transitions should also be present in that theory.

\subsection{Comments on the nature of the transition}

The nature of the transition between these phases depends on microscopic details, and it is not immediately clear how to study the strongly coupled physics when $\bar{\lambda} \sim \mathcal{O}(1)$. One exciting possibility is that the phase transition is continuous, which would exist beyond the Landau paradigm since it separates two distinct topological orders. Furthermore, this would imply the existence of an unstable conformal field theory (CFT) fixed point, which we expect would be quite exotic and perhaps involve emergent symmetries. In the spirit of universality, we attempt here to write down the simplest possible theory of such a transition. The theory we find consists of $N_{f}=4$ flavors of electrically neutral Dirac fermions, $\xi_{i}$, coupled to a $S U(2)_{-1}^{\text {spin }}$ Chern-Simons gauge field, $c$,

$$
\mathcal{L}=\sum_{i=1}^{N_{f}=4} \bar{\xi}_{i}\left(i \not D_{c}-m_{\xi}\right) \xi_{i}-\frac{1}{4 \pi} \operatorname{Tr}\left[c d c-\frac{2 i}{3} c^{3}\right]-\frac{3}{2} \frac{1}{4 \pi} A d A
$$

We emphasize that the fields $\xi$ and $c$ need not have any local relationship with the fields in Theory A nor Theory B. This theory has a $U(4)$ global symmetry rotating the fermion flavors. For $m_{\xi} \gg 0$, the theory is in the Abelian, $S U(2)_{+1}^{\text {spin }} \leftrightarrow U(1)_{-2}$ phase, and for $m_{\xi} \ll 0$ the theory is in the non-Abelian, $S U(2)_{-3}^{\text {spin }} \leftrightarrow U(3)_{2}$ phase. While the flavor index can be interpreted as a kind of Landau level index, there is no a priori reason for this symmetry to be enforced, rendering this theory at best a multicritical point. What's more, this theory has $N_{f}>2|k|=2$, meaning that it falls outside of the Chern-Simons-matter dualities of Eqs. (2.1)-(2.3) and may spontaneously break the flavor symmetry at small values of $m_{\xi}$

\footnotetext{
${ }^{6}$ Note that unlike the fermions of Theory B, the magnetic field felt by the $\psi$ fermions depends both on the background chemical potential and magnetic field, so $\omega_{c}$ is not precisely the Landau level gap, which is determined by $\left\langle\varepsilon^{i j} \partial_{i} a_{j}\right\rangle$.
} 


\begin{tabular}{||c||c|c|}
\hline$\nu_{*}$ & -1 & $\infty$ \\
\hline$\nu_{\psi}+1 / 2$ & $\infty$ & 2 \\
\hline$\nu_{\chi}+1 / 2$ & 2 & $\infty$ \\
\hline Theory A & Metal & Superfluid \\
\hline Theory B & $U(2)_{2}$ & Metal \\
\hline
\end{tabular}

Table 2: Solutions to Eq. (3.16) in which one of the two dual theories is metallic, i.e. is at infinite filling.

[37]. We therefore find the presence of a direct second-order transition unlikely, and we conjecture that any other possible CFT with these two phases also has an enlarged set of global symmetries compared to the underlying UV problem. ${ }^{7}$ We also conjecture that this is the case for the other transitions discussed in this work.

Therefore, it is perhaps more natural to expect the mundane scenario in which the two phases are separated by a first order transition. Starting in, say, the $U(1)_{2}$ phase, as $\bar{\lambda}$ is increased, phase separation will set in, yielding bubbles and stripes of the $U(3)_{2}$ phase, which eventually fill the system. It is also possible that the transition is not direct, and that several different phases arise when $\bar{\lambda} \sim \mathcal{O}(1)$. We cannot exclude this possibility. That we cannot present a thorough description of the transition is not surprising, given the complexity of the phase diagrams for electrons in magnetic fields at fractional filling.

\subsection{Non-Abelian duality and paired FQH phases}

In the above subsection, we conspicuously left out a discussion of the case when the $\chi$ composite fermions fill two Landau levels, corresponding to filling $\nu_{*}=1$. At this filling, Eqs. (3.4) and (3.5) indicate that the $\psi$ composite fermions of Theory $\mathbf{A}$ feel a vanishing magnetic field and therefore form a metallic state with density set by the background magnetic field, $\left\langle\psi^{\dagger} \psi\right\rangle=B / 2 \pi$. This is the well known metallic, composite Fermi liquid state of bosons with unit filling [44]. On the other hand, the analysis of Section 3.2 finds Theory B in an IQH state, yielding $U(2)_{2}$ topological order. Although one of the predicted phases is gapless, we nevertheless propose that the same scenario presented above holds in this theory: the ultimate choice of ground state is determined by the order in which the IR and $\omega_{c} \rightarrow \infty$ limits are taken. These states are again separated by a phase transition, which is tuned by

\footnotetext{
${ }^{7}$ While one may also wish to consider theories with bosonic matter, we note that it is not possible to condense bosonic operators to transition between $U(3)_{2}$ and $U(1)_{-2}$ due to the difference in the signs of the level. This cannot be repaired with level-rank duality because the $U(1)_{-2}$ gauge field is not spin.
} 
the dimensionless coupling of the kind defined in Eq. (3.18).

In contrast to the cases in which both phases were gapped, here we can clearly understand this transition in terms of the $\psi$ composite fermions of Theory $\mathbf{A}$. Indeed, the $U(2)_{2}$ state can be shown to be one of a range of non-Abelian phases of bosons at $\nu=1$ that can be obtained as paired states of the composite Fermi liquid [45], the most famous of which being the $S U(2)_{2}$ bosonic Pfaffian state [13]. The major difference between the $U(2)_{2}$ state and the $S U(2)_{2}$ state lies in the topological spin of the non-Abelian half-vortex, which is set by the pairing momentum channel. As we will explain in more detail in the following subsection, the appropriate channel to obtain the $U(2)_{2}$ order is $l=2$. Consequently, this transition can be understood in terms of the flow of the pairing interaction, which is a four-fermion operator in the Abelian Theory A. Interestingly, it is expected from numerical simulations and recent analytic calculations that the composite Fermi liquid state of bosons at $\nu=1$ is unstable to pairing in the lowest Landau level limit $[46,47]$, perhaps suggesting that the metallic state seen in Theory A has no basin of attraction unless the theory is modified in some way.

From the point of view of Theory $\mathbf{B}$, it is natural to expect that $\bar{\lambda}$ is again the correct running coupling, with the choice of ground state again being viewed as a question of the order in which the lowest Landau level and IR limits are taken. As above, for $\bar{\lambda}>\bar{\lambda}_{*}$, the $\chi$ fermions find themselves in an IQH state, yielding the $U(2)_{2}$ topological order. For $\bar{\lambda}<\bar{\lambda}_{*}$, this picture breaks down, leading to a theory involving bosonic baryons, which we conjecture form the $\nu=1$ metallic state.

Remarkably, at filling $\nu_{*}=1$, we have observed a duality between composite fermion pairing in Theory A and the IQH effect in Theory B. This is surprising, as there is no known dictionary of local operators that makes this connection explicit. Indeed, while the $U(2)_{2}$ state has previously been obtined both in the Read-Green pairing picture [12, 13] and as an IQH state of non-Abelian partons [7, 30, 31, 48], it has never before been suggested that these two constructions may be dual to one another. We will see below that this duality is not limited to the particular case of bosons at $\nu=1$ : in Section 3.6, we will encounter a parallel story involving the anti-Pfaffian state of fermions at $\nu=1 / 2$.

Similar competing ground states are found as the external magnetic field is turned off, i.e. $\nu_{*} \rightarrow \infty$ (see Table 2). In this case, the analysis of Section 3.2 indicates that Theory A predicts a superfluid state, the usual fate of bosons at finite density and $B=0$. On the other hand, the composite fermions of Theory $\mathbf{B}$ form a metal with density equal to the background charge density, a far more exotic non-Fermi liquid state which surely requires that the fundamental bosonic charges be very strongly interacting. Nevertheless, 
the interpretation of the transition between these states is natural from the point of view of Theory B: again adopting the notation of Section 3.3, for $\bar{\lambda}>\bar{\lambda}_{*}$, the theory remains metallic, while for $\bar{\lambda}<\bar{\lambda}_{*}$, the $\chi$ fermions confine to form bosonic baryons. Since these bosons feel no magnetic field, they would then condense and spontaneously break $U(1)_{\mathrm{EM}}$, forming the superfluid state seen in Theory A. Given how natural the state predicted in Theory A is, one might wonder if the metallic state predicted by Theory B is ever stable to baryon condensation. We leave this question for future work.

\subsection{Examples in other fermion-fermion dualities}

Thus far, we have focused our analysis on the dual fermionic theories appearing in the $S U(2)$ quadrality. However, the above considerations are broadly applicable to any pair of fermionic theories related by a Chern-Simons matter duality. To illustrate this point, we consider a more general composite fermion duality describing a transition between the $\nu=1 / k$ Laughlin state and an insulator. This duality relates a theory of Dirac composite fermions with $k-1$ (Abelian) fluxes attached (Theory $\mathbf{A}^{\prime}$ ) to a theory of composite fermions coupled to a $U(N)$ gauge field (Theory B') [49],

$$
\begin{gathered}
\mathcal{L}_{A^{\prime}}(k)=i \bar{\psi} \not D_{a} \psi-\frac{1}{2} \frac{1}{4 \pi} a d a+\frac{1}{2 \pi} a d v+\frac{k-1}{4 \pi} v d v+\frac{1}{2 \pi} v d A+\ldots \\
\qquad \\
\mathcal{L}_{B^{\prime}}(k, N)=i \bar{\eta} \not D_{u} \eta-\frac{1}{2} \frac{1}{4 \pi} \operatorname{Tr}\left[u d u-\frac{2 i}{3} u^{3}\right]-\frac{N-k}{4 \pi} b d b-\frac{1}{2 \pi} \operatorname{Tr}[u] d b+\frac{1}{2 \pi} b d A+\ldots
\end{gathered}
$$

In Theory $\mathbf{A}^{\prime}, \psi$ is a Dirac fermion charged under an emergent $U(1)$ gauge field $a$, and $v$ is another $U(1)$ gauge field. In Theory $\mathbf{B}^{\prime}, \eta$ is a Dirac fermion in the fundamental representation of $U(N), u$ is a $U(N)$ gauge field, and $b$ is a $U(1)$ gauge field. These dualities are derived in Appendix B.1, where it is also shown that these theories are dual composite fermion descriptions of the bosonic Landau-Ginzburg theory for the $\nu=1 / k$ Laughlin state: when $k$ is even, the fundamental charges are bosons, while when $k$ is odd they are fermions (note that when $N=k=2$, we recover Theory $\mathbf{A}$ and Theory $\mathbf{B}$, which are associated with the $\nu=1 / 2$ bosonic Laughlin state). We emphasize that the above duality holds regardless of the rank $N$ of the gauge group $U(N)$ in Theory $\mathbf{B}^{\prime}$, and hence amounts to the statement that $\mathcal{L}_{A^{\prime}}(k)$ is dual to an infinite number of theories $\mathcal{L}_{B^{\prime}}(k, N)$ parameterized by the integer $N$.

As in the examples encountered thus far, we find special filling fractions, $\nu_{*}$, at which 


\begin{tabular}{||c||c|c|c|c||}
\hline$\nu_{*}$ & $1 /(k-1)$ & $1 /(k-2)$ & $3 /(3 k-4)$ & $2 /(2 k-3)$ \\
\hline$\nu_{\psi}+1 / 2$ & $\infty$ & 2 & 4 & 3 \\
\hline$\nu_{\eta}+1 / 2$ & 2 & $\infty$ & 3 & 4 \\
\hline Theory $\mathbf{A}^{\prime}$ & Metal & $\begin{array}{c}U(1)_{k-2}(k \neq 2) \\
\text { Superfluid }(k=2)\end{array}$ & Jain & Jain \\
\hline Theory $\mathbf{B}^{\prime}(N=2)$ & $U(2)_{2,2(k-1)}$ & Metal & $U(3)_{2,3 k-4}$ & $U(4)_{2,2(2 k-3)}$ \\
\hline
\end{tabular}

Table 3: Fillings at which one of Theory $\mathbf{A}^{\prime}$, Eq. (3.20), and Theory $\mathbf{B}^{\prime}$, Eq. (3.21), predicts a metallic ground state and the other a nonmetallic state (first two columns), or where both predict distinct topological orders (last two columns). Here, $N$ is the rank of the $U(N)$ gauge group in Theory $\mathbf{B}^{\prime}$, which is always equal to two in these examples. By Jain we mean a $U(1)^{\mathrm{spin}} \times U(1)$ theory describing the usual Abelian Jain state at filling $\nu_{*}$.

the dual theories predict differing ground states. The details of this analysis are essentially identical to that of the preceding $S U(2)$ examples and are presented in detail in Appendix B.2. Our results are summarized in Table 3. For example, at the filling $\nu_{*}=2 /(2 k-3)$, Theory $\mathbf{A}^{\prime}$ predicts the usual Abelian Jain state, while Theory $\mathbf{B}^{\prime}$ predicts the more exotic, non-Abelian $U(4)_{2,2(2 k-3)}$ state. Our dynamical proposal for understanding the transitions between these states, as well as the others featured in Table 3, is essentially identical to that of Section 3.3, and so we will not comment on it further.

One state of particular note is the $U(3)_{2,-1}$ topological order, which is level-rank dual to $U(2)_{-3,-1}^{\text {spin }}$, predicted by Theory $\mathbf{B}^{\prime}$ with $k=1$ at $\nu_{*}=-3$. Now, the non-spin $U(2)_{3,1}$ ChernSimons theory is dual to the (also non-spin) $\left(G_{2}\right)_{1}$ Chern-Simons theory [50]. Remarkably, $\left(G_{2}\right)_{1}$ describes precisely the Fibonacci topological order, which supports a single non-trivial anyon, $\tau$, obeying the fusion rule $\tau \times \tau=1+\tau$, and is of particular import from the perspective of topological quantum computation [34]. It is rather interesting that Theory $\mathbf{B}^{\prime}$ predicts the (spin) Fibonacci topological order to appear in competition with the $\nu_{*}=-3$ IQH state. In future work, we will explore how other mechanisms can lead to the emergence of this exotic order, using bosonic theories, in the spirit of our earlier construction [15].

Additionally, we wish to highlight the cases at fillings $\nu_{*}=1 /(k-1)$, in which the nonAbelian $U(2)_{2,2(k-1)}$ state predicted by Theory $\mathbf{B}^{\prime}$ can again be understood as a pairing instability of the Abelian composite Fermi liquid state in Theory $\mathbf{A}^{\prime}$. The argument that $U(2)_{2,2(k-1)}$ can be obtained from pairing in Theory $\mathbf{A}^{\prime}$ parallels that for the $U(2)_{2}$ state discussed in the previous subsection, which corresponds to the special case $k=2$. Indeed, the non-Abelian part of $U(2)_{2,2(k-1)}$ is again $S U(2)_{2}$, and the major difference from this state lies in the topological spins of the non-Abelian half vortices, which are modified by the level 
of the Abelian sector. Moreover, one can check explicitly that pairing of the Theory $\mathbf{A}^{\prime}$ composite fermions in the $l=2$ angular momentum channel yields the expected $U(2)_{2,2(k-1)}$ topological order for all $k$ by considering the edge spectrum: this topological order supports three chiral, charge-neutral Majorana fermions and one anti-chiral $U(1)_{k-1}$ bosonic charge mode. From the point of view of Theory $\mathbf{A}^{\prime}, l=2$ pairing leads to the three chiral Majorana fermions, in addition to a $U(1)_{k-1}$ charge mode coming from the left over Abelian sector. That such a large class of non-Abelian topological orders which can be understood via pairing in a composite fermion theory, in this case Theory $\mathbf{A}^{\prime}$, has a dual description as IQH states of composite fermions in a dual non-Abelian theory, Theory $\mathbf{B}^{\prime}$ is quite remarkable.

We close this section by commenting on some particular examples of physical interest. First, for $k=1$ and $\nu_{*}=\infty$, Theory $\mathbf{A}^{\prime}$ is a free Dirac fermion at finite chemical potential but vanishing magnetic field. In this case, in Theory $\mathbf{B}^{\prime}$, it is possible to integrate out the auxiliary gauge field $b$ without violating flux quantization: its Chern-Simons level is $-(N-k)=-1$. This cancels the Chern-Simons term for the Abelian part of the $U(2)$ gauge field, $\operatorname{Tr}[u]$, Higgsing the background EM field, $A_{\mu}$, and leading to a chiral superconductor with topological order is $U(2)_{2,0}=\left[S U(2)_{2} \times U(1)_{0}\right] / \mathbb{Z}_{2} \cong S O(3)_{1}$, which is Abelian. This state contains only a single anyon, a Majorana fermion ${ }^{8}[26,51]$. Such a state can be accessed via a pairing instability in Theory $\mathbf{A}^{\prime}$. It is natural to expect this instability to arise due to the effects of local four-fermion operators that destabilize Theory $\mathbf{A}^{\prime}$ in the order of limits in which the $\eta$ fermions of Theory $\mathbf{B}^{\prime}$ form an IQH state.

For $k=-1$ and $\nu_{*}=-1 / 2$, Theory $\mathbf{A}^{\prime}$ is Son's Dirac composite Fermi liquid theory of the half-filled Landau level [52]. In this case, the topological order predicted by Theory $\mathbf{B}^{\prime}$ is $U(2)_{2,-4}=\left[S U(2)_{2} \times U(1)_{-8}\right] / \mathbb{Z}_{2}$, which is the (time-reversed) topological order of the famous anti-Pfaffian state, another paired state of composite fermions [35, 36]! Additionally, for $k=3$ and $\nu_{*}=1 / 2$, Theory $\mathbf{B}^{\prime}$ predicts the $\left[S U(2)_{2} \times U(1)_{8}\right] / \mathbb{Z}_{2}=U(2)_{2,4}$ order, which is that of Wen's (221) parton state [7,30], another proposed ground state of fermions at half filling that can be understood in terms of pairing in Theory $\mathbf{A}^{\prime}$, which now has additional attached fluxes. Now, although these states are all accessible within parton constructions (see, for example, Ref. [48]), we must again emphasize that the projective framework hinges on the dynamical assumption that the electron fractionalizes into partons which do not confine. Finally, another non-trivial bosonic example is $k=0$ and $\nu_{*}=1$, in which Theory $\mathbf{B}^{\prime}$ predicts a $\left[S U(2)_{2} \times U(1)_{-4}\right] / \mathbb{Z}_{2}=U(2)_{2,-2}$ ground state, which describes the Ising topological order [53]. While these results are reminiscent of the parton constructions giving

\footnotetext{
${ }^{8}$ This can be viewed as a consequence of the condensation of the Majorana fermion of the $S U(2)_{2}$ topological order, which confines the non-Abelian "half-vortex" of the $S U(2)_{2}$ factor.
} 
these states, the use of duality provides a connection between partonic intuition and the dynamics of pairing. Additionally, it is straightforward to check in these examples that $l=2$ pairing in Theory $\mathbf{A}^{\prime}$ yields the expected $U(2)_{2,2(k-1)}$ order by comparing the edge theories. It would be interesting to determine if other dual descriptions exist which yield the other proposed Pfaffian-like states, which arise from pairing the composite fermions of Theory $\mathbf{A}^{\prime}$ in other angular momentum channels, as IQH phases of dual composite fermions, and we leave this to future work.

\section{Building non-Abelian states from excitonic pairing}

In the preceding section, we illustrated how non-Abelian Chern-Simons matter dualities may be used to map out parts of the phase diagram for electrons (or bosons) in a magnetic field at certain fractional fillings, $\nu_{*}$, finding gapless states as well as both Abelian and non-Abelian topological orders. We now turn to present an alternative means of constructing non-Abelian FQH states, still making use of the dual composite fermion theories employed above. Our goal here is to provide a complementary perspective to our previous work [15], in which we constructed Landau-Ginzburg theories for the Read-Rezayi states using bosonic ChernSimons-matter using a multilayer pairing procedure. Specifically, we will consider condensing interlayer excitons (pairs of fermions in different layers which are neutral under the external EM gauge field) in Theory B, Eq. (3.2), to generate non-Abelian states. We will find, however, that the excitonic paired phases are not the Read-Rezayi states constructed in Ref. [15], but rather the Blok-Wen states with $U(k)_{2}$ topological order, as in Section 3.

For simplicity, we again consider a bilayer system, with each layer being a $\nu=1 / 2$ bosonic Laughlin state. We use the dual fermionic description of Theory $\mathbf{B}$ for each layer so that the (initially decoupled) multilayer system is described by the Lagrangian

$$
\mathcal{L}_{B, 2}=\sum_{n=1}^{2}\left(i \bar{\chi}_{n} \not D_{b_{n}-A \mathbf{1} / 2} \chi_{n}-\frac{1}{2} \frac{1}{4 \pi} \operatorname{Tr}\left[b_{n} d b_{n}-\frac{2 i}{3} b_{n}^{3}\right]\right)-\frac{1}{2} \frac{1}{4 \pi} A d A
$$

Here, $\chi_{n}$ and $b_{n}$ are the composite fermions and $S U(2)$ gauge fields on layer $n$, respectively. Note that each layer couples in the same way to the external electromagnetic field, $A$. Moreover, we see from Table 1 that when each layer is at filling $\nu=1 / 2$, so that the full multilayer system is at filling $\nu=1$, the $\chi_{n}$ of each layer form an IQH state.

Now, following the standard approach [54], we introduce an interlayer excitonic pairing interaction mediated by an electrically neutral scalar field, $\Sigma$,

$$
\mathcal{L}_{\text {exciton }}=\bar{\chi}_{1} \Sigma \chi_{2}+\text { H.c. }
$$


The field $\Sigma$ can be thought of as a Hubbard-Stratonovich field for the pairing interaction. It couples minimally to the gauge fields on either layer, and so has dynamics described by

$$
\mathcal{L}_{\Sigma}=\left|\partial \Sigma-i b_{1} \Sigma+i \Sigma b_{2}\right|^{2}-V[\Sigma],
$$

where $V[\Sigma]$ is the potential for $\Sigma$. Under gauge transformations,

$$
\Sigma \mapsto U_{1} \Sigma U_{2}^{\dagger}, \quad U_{m} \in S U(2) \text { on layer } m \text {. }
$$

The full multi-layer theory is therefore described by the Lagrangian,

$$
\mathcal{L}_{\text {bilayer }}=\mathcal{L}_{B, 2}+\mathcal{L}_{\text {exciton }}+\mathcal{L}_{\Sigma}
$$

The condensation of $\Sigma$ yields the excitonic paired state, characterized by a non-zero expectation value for the operator $\bar{\chi}_{1} \chi_{2}$. It should be emphasized that in the dual descriptions of Theory A and the Landau-Ginzburg theory of Eq. (2.5), the interaction $\mathcal{L}_{\text {exciton }}$ will correspond to a highly nonlocal object involving monopole operators. In general, the fundamental fields do not map to local operators under the dualities of Eq. (2.1)-(2.3) [16]. Hence, the upshot of examining this dual fermionic theory is that we can access regions of the phase diagram at a given filling fraction, which are less readily understood in the formulation of Theory A or the original bosonic Landau-Ginzburg theory, as they would require the inclusion of complicated, nonlocal interactions.

Now, suppose we have a nonzero magnetic field such that each layer is at filling $\nu=1 / 2$, meaning the $\chi_{n}$ each fill a single Landau level, as indicated in Table 1. We assume that we can safely integrate out the occupied $\chi_{n}$ Landau levels, yielding additional level $-1 / 2$ Chern-Simons terms for the $b_{m}$ gauge fields. The effective action describing this gapped state is then

$$
\mathcal{L}_{\text {bilayer }}=-\sum_{n=1}^{2} \frac{1}{4 \pi} \operatorname{Tr}\left[b_{n} d b_{n}-\frac{2 i}{3} b_{n}^{3}\right]-\frac{1}{4 \pi} A d A+\tilde{\mathcal{L}}_{\Sigma}+\ldots,
$$

where we have integrated out the fermions $\chi$, leading to a renormalized Lagrangian for $\Sigma$, denoted $\tilde{\mathcal{L}}_{\Sigma}$. If the potential $V[\Sigma]$ is such that the field $\Sigma$ is massive and does not condense, then we are simply left with an $S U(2)_{-1}^{\text {spin }} \times S U(2)_{-1}^{\text {spin }} \leftrightarrow U(1)_{2} \times U(1)_{2}$ Chern-Simons theory at low energy, describing two decoupled layers of $\nu=1 / 2$ Laughlin states. Now, suppose instead that the potential $V[\Sigma]$ is such that $\Sigma$ obtains a non-zero vacuum expectation value, $\langle\Sigma\rangle \propto 1$. In this excitonic paired state, the gauge group $S U(2) \times S U(2)$ will be Higgsed down to the diagonal $S U(2)$ subgroup, as follows from the gauge transformations of Eq. 
(4.4). Explicitly, from the Lagrangian for $\Sigma$, the linear combination $b_{1}-b_{2}$ of the gauge fields acquires a mass, effectively identifying the gauge fields of each layer: $b_{1} \equiv b_{2} \equiv b$. Hence, the Chern-Simons terms will add, resulting in a non-Abelian $S U(2)_{-2}^{\text {spin }} \leftrightarrow U(2)_{2}$ Chern-Simons theory at low energies.

It is possible to explicitly write out the anyon spectrum of this $U(2)_{2}$ topological order in terms of composite operators of the fundamental fermions. This requires identifying the operators which transform under the non-trivial spin- $1 / 2$ and spin- 1 representations of the $S U(2)_{-2}$ gauge theory and taking into account additional spin factors coming from the underlying fermionic statistics of said operators. For reference, we denote the anyons transforming in the spin-1/2 and spin-1 representations of the $S U(2)_{-2}$ topological order as $\sigma$ and $\psi$ (not to be confused with the fermion field in Theory A), corresponding to the non-Abelian half-vortex (i.e. the Ising twist field) and Abelian Majorana fermion, respectively, in the time-reversed conjugate of the bosonic $\nu=1$ Moore-Read state. They have $\operatorname{spin}^{9} h_{\sigma}=-3 / 16$ and $h_{\psi}=1 / 2$, respectively, and satisfy the fusion rules $\sigma \times \sigma=1+\psi$ and $\psi \times \psi=1$, where 1 represents the vacuum. Now, in our theory, the minimal charge anyons are represented by the fermions $\chi_{1}$, which transform in the fundamental (or spin-1/2) representation of $S U(2)_{-2}$. The $\chi_{1}$ operators will thus satisfy the same fusion rules as the $\sigma$ anyon in the $S U(2)_{-2}$ theory, but they have spin $-3 / 16+1 / 2=5 / 16$, due to the bare fermionic statistics of $\chi_{1}$. The other non-trivial anyon is represented by the composite operator $\bar{\chi}_{1} \tau^{a} \chi_{1}$, where $\tau^{a}$ is the vector of generators of $S U(2)$. This operator is charge neutral and transforms in the spin-1 representation of $S U(2)$, meaning it obeys the same fusion rules as $\psi$. It also has the same spin as $\psi, h_{\psi}=1 / 2$, since it has bare bosonic statistics, being a bilinear in fermion operators. Once can check that these anyons with these spins match the anyon spectrum expected for the $U(2)_{2}$ topological order. Finally, note that the fundamental fermions $\chi_{1}$ and $\chi_{2}$ are indistinguishable in the excitonic paired phase, as one can be transmuted into the other via the the $\left\langle\bar{\chi}_{1} \chi_{2}\right\rangle$ condensate. Hence, there is no double-counting of anyons.

Several remarks on this construction are in order. This excitonic pairing mechanism is somewhat unconventional and differs from the more common Read-Green construction [12] used to describe the Moore-Read states. In the latter picture, the electrons (or bosons) are mapped to composite fermions using non-relativistic flux attachment. At the appropriate filling fractions, the composite fermions see an effectively vanishing flux at mean-field level. The resulting composite Fermi liquid can give way to a pairing instability in the $p+i p$ channel,

\footnotetext{
${ }^{9}$ Here, the spin of an anyon $a$ is the phase factor $\exp \left(2 \pi i h_{a}\right)$ picked up when rotating it through an angle of $2 \pi$. This is not to be confused with the spin- $j / 2$ representations of $S U(2)$.
} 
Higgsing the dynamical $U(1)$ Chern-Simons gauge field down to its $\mathbb{Z}_{2}$ subgroup and resulting in a gapped state. The non-Abelian Ising anyons in the Moore-Read state then have a description in terms of vortices of the Chern-Simons gauge field. In the present construction, we are instead pairing fermions on top of a filled Landau level, a gapped state. Hence, unlike the Read-Green picture, we cannot understand our exciton paired state as arising from some perturbative instability, since interactions must be sufficiently strong to overcome the gap. In addition, one can check from standard homotopy arguments that the symmetry breaking pattern $S U(2) \times S U(2) \rightarrow S U(2)_{\text {diagonal }}$ does not admit vortex configurations [14, 15]. Instead, the anyon spectrum in our model is generated by composite objects formed from the fundamental fermions, as outlined above. It should be noted that even our earlier bosonic construction [15] required a similarly unconventional pairing mechanism, in which it was necessary to assume that composite bosons paired rather than condensed. Finally, it is clear that we can generalize our construction to a multilayer system with $k$ copies of the $\nu=1 / 2$ Laughlin state; interlayer excitonic pairing in such a system would lead to a $U(k)_{2}$ topological order.

\section{Discussion}

Employing non-Abelian composite fermion dualities, we have presented two complementary pictures for describing a broad range of non-Abelian FQH states, which can be obtained either as IQH states of non-Abelian composite fermions or as excitonic states in multilayer systems. Along the way, we developed new insights into the non-Abelian theories' dynamics, in which the order of the lowest Landau level $(B \rightarrow \infty)$ and IR limits was seen to play a crucial role in determining the ultimate choice between the non-Abelian ground state and a competing Abelian state that is natural in a dual description. This subtlety has thus far received little attention in studies of non-Abelian dualities, yet we find it to be a ubiquitous feature of non-Abelian fermion-fermion dualities. It may be a worthwhile endeavour to see whether studying these theories at finite magnetic field in the 't Hooft limit, in the vein of Ref. [55], may provide an analytical handle on the physics of these transitions - we leave this for future work. Interestingly, related physics has been observed recently in numerics, where it has been argued that the ground state at certain fillings can exhibit effectively Abelian topological order for short-range interactions and non-Abelian order as the interaction range is increased $[56,57]$. We hope that our work will motivate more numerical efforts in this direction.

Although we cannot make many concrete statements about the transitions we propose to 
occur between the Abelian and non-Abelian states, we remarkably find several examples in which the non-Abelian states - among them the anti-Pfaffian - can be understood in terms of pairing in a dual composite Fermi liquid description. Such dualities between composite fermion pairing and the IQH effect in a dual, non-Abelian theory are new, and finding new examples of such dualities will be a fruitful direction for future work. Looking forward, a natural question to ask is whether non-Abelian fermion-fermion dualities can be used to derive other non-Abelian FQH states, beyond the variations of the Blok-Wen states we find. Indeed, although the anti-Pfaffian is a member of the $U(2)_{2,2(k-1)}$ series of states, we do not seem to arrive at the Pfaffian or PH-Pfaffian states. To that end, it may be fruitful to apply our analysis using dualities involving Chern-Simons-matter theories with gauge groups other than $S U(N)$ or $U(N)$, as the family of Pfaffian states can naturally be described using $O(2)_{2, L}$ Chern-Simons theories [58]. It is also somewhat peculiar in that the Read-Rezayi and generalized non-Abelian spin singlet states, which are readily obtained through non-Abelian bosonic theories, do not appear to be accessible within the present approach. We leave the construction of fermionic theories for these states to future work. Conversely, we found that the (spin) Fibonacci topological order appears naturally in Sec. 3.6 within this composite fermion approach. It would be interesting to see whether a variation of our earlier bosonic construction [15] may allow for accessing this exotic state as well.

\section{Acknowledgements}

We thank Gil Young Cho, Michael Mulligan, and Chong Wang for useful discussions. This work was supported in part by the National Science Foundation under Grant No. DMR1725401 at the University of Illinois (EF, HG, RS). RS also acknowledges the support of the Natural Sciences and Engineering Research Council of Canada (NSERC) [funding reference number 6799-516762-2018]. HG also acknowledges support from the NSF Graduate Research Fellowship Program under Grant No. DGE-1144245 and from the Gordon and Betty Moore Foundation EPiQS Initiative through Grant No. GBMF8684 at the Massachusetts Institute of Technology.

\section{A Chern-Simons Conventions}

In this appendix, we lay out our conventions for non-Abelian Chern-Simons gauge theories. We define $U(N)$ gauge fields $a_{\mu}=a_{\mu}^{b} t^{b}$, where $t^{b}$ are the (Hermitian) generators of the Lie algebra of $U(N)$, which satisfy $\left[t^{a}, t^{b}\right]=i f^{a b c} t^{c}$, where $f^{a b c}$ are the structure constants of 
$U(N)$. The generators are normalized so that $\operatorname{Tr}\left[t^{b} t^{c}\right]=\frac{1}{2} \delta^{b c}$. The trace of $a$ is a $U(1)$ gauge field, which we require to satisfy the Dirac quantization condition,

$$
\int_{\Sigma} \frac{d \operatorname{Tr}[a]}{2 \pi}=n \in \mathbb{Z}
$$

where $\Sigma \subset X$ is an oriented 2-cycle in spacetime, which we denote $X$. If $a_{\mu}$ couples to fermions, then it is a $\operatorname{spin}_{c}$ connection, and it satisfies a modified flux quantization condition

$$
\int_{\Sigma} \frac{d \operatorname{Tr}[a]}{2 \pi}=\int_{\Sigma} \frac{w_{2}}{2}+n, n \in \mathbb{Z},
$$

where $w_{2}$ is the second Stiefel-Whitney class of $X$. In general, the Chern-Simons levels for the $S U(N)$ and $U(1)$ components of $a$ can be different. We therefore adopt the standard notation [16],

$$
U(N)_{k, k^{\prime}}=\frac{S U(N)_{k} \times U(1)_{N k^{\prime}}}{\mathbb{Z}_{N}}
$$

By taking the quotient with $\mathbb{Z}_{N}$, we are restricting the difference of the $S U(N)$ and $U(1)$ levels to be an integer multiple of $N$,

$$
k^{\prime}=k+n N, n \in \mathbb{Z} .
$$

This enables us to glue the $U(1)$ and $S U(N)$ gauge fields together to form a gauge invariant theory of a single $U(N)$ gauge field $a=a_{S U(N)}+\tilde{a} \mathbf{1}$, with $\operatorname{Tr}[a]=N \tilde{a}$ having quantized fluxes as in Eq. (A.1). The Lagrangian for the $U(N)_{k, k^{\prime}}$ theory can be written as

$$
\mathcal{L}_{U(N)_{k, k^{\prime}}}=\frac{k}{4 \pi} \operatorname{Tr}\left[a_{S U(N)} d a_{S U(N)}-\frac{2 i}{3} a_{S U(N)}^{3}\right]+\frac{N k^{\prime}}{4 \pi} \tilde{a} d \tilde{a} .
$$

For the case $k=k^{\prime}$, we simply refer to the theory as $U(N)_{k}$.

Throughout this paper, we implicitly regulate non-Abelian (Abelian) gauge theories using Yang-Mills (Maxwell) terms, as opposed to dimensional regularization [2, 59]. In Yang-Mills

regularization, there is a one-loop exact shift of the $S U(N)$ level, $k \rightarrow k+\operatorname{sgn}(k) N$, that does not appear in dimensional regularization. Consequently, to describe the same theory in dimensional regularization, one must start with a $S U(N)$ level $k_{\mathrm{DR}}=k+\operatorname{sgn}(k) N$. The dualities discussed in this paper, e.g. Eqs. (2.1)-(2.3), therefore would take a somewhat different form in dimensional regularization.

\section{B Details of $U(N)$ Fermion-Fermion Duality Examples}

In this Appendix we provide the details of the analysis outlined in Section 3.6. We begin by deriving the duality between Eq. (3.20) and Eq. (3.21) and then identify the states listed in Table 3 . 


\section{B.1 Derivation of the Duality}

As noted in Section 3.6, the fermionic theories Theory $\mathbf{A}^{\prime}$ and Theory $\mathbf{B}^{\prime}$ are both dual to the bosonic Landau-Ginzburg theory for the $\nu=1 / k$ Laughlin state, which is described by the Lagrangian

$$
\mathcal{L}_{\Phi}(k)=\left|D_{b} \Phi\right|^{2}-|\Phi|^{4}+\frac{k}{4 \pi} b d b+\frac{1}{2 \pi} b d A .
$$

Here, $\Phi$ is a complex scalar field, $b$ is a $U(1)$ gauge field, and $A$ is the external electromagnetic field. It is straightforward to see that one obtains the Laughlin state when $\Phi$ is gapped by a mass and a trivial insulator when $\Phi$ condenses.

In order to derive these dualities, we take as our starting point the $S U / U$ duality of Eq. $(2.2)$,

$$
\left|D_{A} \Phi\right|^{2}-|\Phi|^{4} \longleftrightarrow i \bar{\eta} \not D_{u} \eta-\frac{1 / 2}{4 \pi} \operatorname{Tr}\left[u d u-\frac{2 i}{3} u^{3}\right]-\frac{1}{2 \pi} \operatorname{Tr}[u] d A-\frac{N}{4 \pi} A d A, \quad a \in U(N),
$$

where $u$ is a $U(N)$ gauge field and $\eta$ is a fermion in the fundamental representation of $U(N)$. Note that the rank $N$ can be an arbitrary integer, and so the above equation implies that the Wilson-Fisher theory is dual to an infinite number of fermionic $U(N)$ gauge theories. Now, one can derive new dualities from old ones by applying the modular transformations $[60]$

$$
\mathcal{S}: \mathcal{L}[A] \mapsto \mathcal{L}[b]+\frac{1}{2 \pi} A d b, \quad \mathcal{T}: \mathcal{L}[A] \mapsto \mathcal{L}[A]+\frac{1}{4 \pi} A d A
$$

to both sides of a duality, where again $A$ is the background EM field and $b$ is a new dynamical $U(1)$ gauge field. Here, $\mathcal{S}$ is the operation of promoting a background gauge field to a dynamical one, while $\mathcal{T}$ corresponds to the addition of a Landau level. Applying $\mathcal{S} \mathcal{T}^{k}$ to the $S U / U$ duality yields,

$$
\mathcal{L}_{\Phi}(k) \longleftrightarrow i \bar{\eta} \not D_{u} \eta-\frac{1 / 2}{4 \pi} \operatorname{Tr}\left[u d u-\frac{2 i}{3} u^{3}\right]-\frac{1}{2 \pi} \operatorname{Tr}[u] d b-\frac{N-k}{4 \pi} b d b+\frac{1}{2 \pi} b d A=\mathcal{L}_{B^{\prime}}(k, N) .
$$

On the other hand, we can also consider the Abelian bosonization duality [26, 27],

$$
\left|D_{A} \Phi\right|^{2}-|\Phi|^{4} \longleftrightarrow i \bar{\psi} \not D_{a} \psi-\frac{1}{2} \frac{1}{4 \pi} a d a+\frac{1}{2 \pi} a d A-\frac{1}{4 \pi} A d A
$$

where $a$ is an emergent $U(1)$ gauge field and $\psi$ a Dirac fermion. Applying $\mathcal{S T}^{k}$ to this duality, we find

$$
\mathcal{L}_{\Phi}(k) \longleftrightarrow i \bar{\psi} \not D_{a} \psi-\frac{1}{2} \frac{1}{4 \pi} a d a+\frac{1}{2 \pi} a d v+\frac{k-1}{4 \pi} v d v+\frac{1}{2 \pi} v d A=\mathcal{L}_{A^{\prime}}(k) .
$$


We thus arrive at the desired dualities

$$
\mathcal{L}_{A^{\prime}}(k) \longleftrightarrow \mathcal{L}_{\Phi}(k) \longleftrightarrow \mathcal{L}_{B^{\prime}}(k, N)
$$

We emphasize that these dualities hold true for any value of the rank, $N>0$, of the gauge group $U(N)$ of Theory $\mathbf{B}^{\prime}$.

\section{B.2 Examples involving gapless states}

Let us now investigate the states predicted by the dual theories, Theory $\mathbf{A}^{\prime}$ and Theory $\mathbf{B}^{\prime}$, at fractional electronic filling fractions, following the logic in our study of the dual fermionic theories in the $S U(2)$ quadrality in Section 3. We define the filling fraction of the $\psi$ composite fermions as

$$
\nu_{\psi}=\frac{2 \pi\left\langle\psi^{\dagger} \psi\right\rangle}{\left\langle\varepsilon^{i j} \partial_{i} a_{j}\right\rangle} .
$$

Using the equations of motion of $\mathcal{L}_{A^{\prime}}$, we find the following relationship between the electronic and $\psi$ filling fractions

$$
\nu_{\psi}=\frac{1}{2}+\frac{1}{-1 / \nu+(k-1)} \Longleftrightarrow \nu=\frac{2 \nu_{\psi}-1}{2(k-1) \nu_{\psi}-k-1} .
$$

As for the composite fermions of the non-Abelian Theory $\mathbf{B}^{\prime}$, to define the $\eta$ filling fraction, we first decompose the $U(N)$ gauge field as $u=u_{S U(N)}+\tilde{u} \mathbf{1}$, where $\mathbf{1}$ is the $N \times N$ identity matrix, $\tilde{u}$ is a $U(1)$ gauge field, and $u_{S U(N)}$ is an $S U(N)$ gauge field. In the presence of a non-zero $U(1)$ flux, $\left\langle\varepsilon^{i j} \partial_{i} \tilde{u}_{j}\right\rangle$, the $\eta$ fermion Landau level degeneracy is given by

$$
d_{L L}=\frac{\left\langle\varepsilon^{i j} \partial_{i} \tilde{u}_{j}\right\rangle A}{2 \pi} \times(\text { color degeneracy }) \times(\text { charge })=\frac{\left\langle\varepsilon^{i j} \partial_{i} \tilde{u}_{j}\right\rangle A}{2 \pi} \times N \times 1=\frac{N\left\langle\varepsilon^{i j} \partial_{i} \tilde{u}_{j}\right\rangle A}{2 \pi} .
$$

Hence, the $\eta$ fermion filling fraction is given by ${ }^{10}$

$$
\nu_{\eta}=-\frac{2 \pi\left\langle\eta^{\dagger} \eta\right\rangle}{N\left\langle\varepsilon^{i j} \partial_{i} \tilde{u}_{j}\right\rangle} .
$$

Using the equations of motion of $\mathcal{L}_{B^{\prime}}$, we find

$$
\nu_{\eta}=-\frac{1}{2}+\frac{N}{1 / \nu+N-k} \Longleftrightarrow \nu=\frac{2 \nu_{\eta}+1}{2(k-N) \nu_{\eta}+(k+N)} .
$$

\footnotetext{
${ }^{10} \mathrm{We}$ add the minus sign for consistency with the definition of the $\chi$ filling fraction $\nu_{\chi}$ of Eq. (3.12) when $N=k=2$.
} 
Let us suppose the $\psi$ fermions fill up an integer number of LLs, so that $\nu_{\psi}=p-1 / 2$. Then, from Eq. (B.9), we have

$$
\nu=\frac{p-1}{(p-1)(k-1)-1}
$$

which is simply the Jain sequence of states.

We are interested in seeing whether a gapped state of the $\psi$ fermions ever corresponds to a metallic state of the $\eta$ fermions (i.e. with $\nu_{\eta} \rightarrow \infty$ ). From Eq. (B.12), we see that the $\eta$ fermions form a metallic state when $1 / \nu=-N+k \in \mathbb{Z}$. We must therefore look for solutions of the equation

$$
k-1-(p-1)^{-1}=-N+k .
$$

The only valid solution with $N>0$ is $(N, p)=(2,2)$. So, when $N=2$ and the $\psi$ fermions fill the $p=2$ Landau level, the $\eta$ fermions form a metallic state. The electronic filling fraction is $\nu_{*}=1 /(k-2)$. At this filling, we can integrate out the $\psi$ fermions in Theory $\mathbf{A}^{\prime}$ to obtain the effective action

$$
\mathcal{L}_{A^{\prime}, \mathrm{eff}}=\frac{k-2}{4 \pi} v d v+\frac{1}{2 \pi} v d A .
$$

We thus have two cases to consider: $k=2$ and $k \neq 2$. When $k=2$, Theory $\mathbf{A}^{\prime}$ yields the usual dual theory for a superfluid (recall that there is an implicit Maxwell term in the action for $c$ ). This is not surprising, as the filling fraction of the electrons (which are bosons for $k=2$ ) is $\nu=\infty$, which is to say they see no magnetic field. Hence, for $k=2$, both Theory $\mathbf{A}^{\prime}$ and Theory $\mathbf{B}^{\prime}$ predict compressible states. In contrast, for $k \neq 2$, Theory $\mathbf{A}^{\prime}$ describes the incompressible $\nu=1 /(k-2)$ Laughlin state, while Theory $\mathbf{B}^{\prime}$ again describes a metallic state of the $\eta$ fermions.

Let us now consider the inverse scenario in which the $\eta$ fermions fill an integer number of LLs, so that $\nu_{\eta}=s-1 / 2$. Hence,

$$
\nu=\frac{s}{s(k-N)+N},
$$

From Eq. (B.9), we see that the $\psi$-fermions are in a metallic state when $1 / \nu=k-1$. This implies

$$
\frac{s-1}{s}=N>0
$$

for which the only solution is $(N, s)=(2,2)$. In this case, the electronic filling fraction is $\nu_{*}=1 /(k-1)$. This simply corresponds to the usual sequence of incompressible states for 
the fermionic ( $k$ odd) and bosonic ( $k$ even) Jain sequences. At these fillings, we can integrate out the $\eta$ fermions in Theory $\mathbf{B}^{\prime}$ to obtain the effective action

$$
\mathcal{L}_{B^{\prime}, \text { eff }}=\frac{-2}{4 \pi} \operatorname{Tr}\left[u d u-\frac{2 i}{3} u^{3}\right]+\frac{k-2}{4 \pi} b d b-\frac{1}{2 \pi} \operatorname{Tr}[u] d b+\frac{1}{2 \pi} b d A,
$$

describing a non-Abelian topological order. Specifically, this is the Lagrangian for the $\left[U(2)_{-2}^{\text {spin }} \times U(1)_{4(k-1)}\right] / \mathbb{Z}_{2} \leftrightarrow\left[S U(2)_{2} \times U(1)_{4(k-1)}\right] / \mathbb{Z}_{2}=U(2)_{2,2(k-1)}$ Chern-Simons theory.

In order to understand how one arrives at this identification of the Lagrangian as that for a quotient theory, let us start with a decoupled $U(2)_{-2}^{\text {spin }} \times U(1)_{4(k-1)}$ Chern-Simons theory:

$$
\mathcal{L}=\frac{-2}{4 \pi} \operatorname{Tr}\left[\hat{u} d \hat{u}-\frac{2 i}{3} \hat{u}^{3}\right]+\frac{4(k-1)}{4 \pi} \hat{b} d \hat{b}+\frac{2}{2 \pi} \hat{b} d A .
$$

Now, taking the $\mathbb{Z}_{2}$ quotient of this theory amounts to declaring that $\hat{u}$ and $\hat{b}$ are no longer "good" gauge fields but the linear combinations

$$
\begin{aligned}
& u=\hat{u}-\hat{b} \mathbf{1} \\
& b=2 \hat{b}
\end{aligned}
$$

are [53]. That is to say, we declare $u$ and $b$ to satisfy the appropriate flux quantization conditions. In more formal terms, taking the $\mathbb{Z}_{2}$ quotient means we gauge the common $\mathbb{Z}_{2}$ one-form symmetry of the $U(2)_{-2}^{\text {spin }}$ and $U(1)_{4(k-1)}$ factors $[53,61]$ (which is to say, we project out all Wilson lines which have non-trivial braiding with respect to the Wilson line generating the $\mathbb{Z}_{2}$ one-form symmetry). Rewriting $\mathcal{L}$ in terms of these gauge fields, we arrive at Eq. (B.18), as desired.

\section{B.3 Examples involving gapped states}

Lastly, we can look for filling fractions at which both the $\psi$ and $\eta$ fermions form IQH states. Setting Eqs. (B.13) and (B.16) equal to one another, we find that this happens when

$$
N=\frac{s}{s-1} \frac{p}{p-1}
$$

The topological orders predicted by Theory $\mathbf{A}^{\prime}$ and Theory $\mathbf{B}^{\prime}$ at these filling fractions are described by, respectively, the low energy actions

$$
\begin{aligned}
& \mathcal{L}_{A^{\prime}, \mathrm{eff}}=\frac{p-1}{4 \pi} a d a+\frac{1}{2 \pi} a d v+\frac{k-1}{4 \pi} v d v+\frac{1}{2 \pi} v d A, \\
& \mathcal{L}_{B^{\prime}, \mathrm{eff}}=-\frac{s}{4 \pi} \operatorname{Tr}\left[u d u-\frac{2 i}{3} u^{3}\right]+\frac{k-2}{4 \pi} b d b-\frac{1}{2 \pi} \operatorname{Tr}[u] d b+\frac{1}{2 \pi} b d A .
\end{aligned}
$$


One integer solution to the above equation is given by $(s, p, N)=(3,4,2)$, corresponding to an electronic filling fraction of $\nu_{*}=3 /(3 k-4)$. Here, Theory $\mathbf{A}^{\prime}$ predicts a $U(1)^{\text {spin }} \times$ $U(1)$ theory (note $a$ is a $\operatorname{spin}_{\text {c }}$ connection while $v$ is a regular gauge field) describing the Abelian Jain state at $\nu_{*}=2 k /(2 k-3)$. Using the same quotient construction as in the previous subsection, we can see that Theory $\mathbf{B}^{\prime}$ describes a $\left[U(2)_{-3}^{\text {spin }} \times U(1)_{3(3 k-4)}\right] / \mathbb{Z}_{3} \leftrightarrow$ $\left[S U(3)_{2} \times U(1)_{3(3 k-4)}\right] / \mathbb{Z}_{3}=U(3)_{2,3 k-4}$ topological order. A second integer solution is given by $(s, p, N)=(4,3,2)$, corresponding to an electronic filling fraction of $\nu_{*}=2 /(2 k-3)$. Theory $\mathbf{A}^{\prime}$ again predicts an Abelian Jain state, whereas Theory $\mathbf{B}^{\prime}$ predicts a non-Abelian $\left[U(2)_{-4}^{\text {spin }} \times U(1)_{8(2 k-3)}\right] / \mathbb{Z}_{4} \leftrightarrow\left[S U(4)_{2} \times U(1)_{8(2 k-3)}\right] / \mathbb{Z}_{4}=U(4)_{2,2(2 k-3)}$ topological order.

\section{References}

[1] F. Wilczek, "Magnetic Flux, Angular Momentum, and Statistics," Phys. Rev. Lett. 48, 1144 (1982).

[2] E. Witten, "Quantum field theory and the Jones polynomial," Commun. Math. Phys. 121, 351-399 (1989).

[3] J. K. Jain, "Composite-fermion approach for the fractional quantum Hall effect," Phys. Rev. Lett. 63, 199 (1989).

[4] A. López and E. Fradkin, "Fractional quantum Hall effect and Chern-Simons gauge theories," Phys. Rev. B 44, 5246 (1991).

[5] S. C. Zhang, T. H. Hansson, and S. Kivelson, "Effective-Field-Theory Model for the Fractional Quantum Hall Effect," Phys. Rev. Lett. 62, 82 (1989).

[6] X.-G. Wen, "Topological orders and edge excitations in fractional quantum hall states," Advances in Physics 44, 405-473 (1995).

[7] X. G. Wen, "Non-abelian statistics in the fractional quantum hall states," Phys. Rev. Lett. 66, 802-805 (1991).

[8] G. Moore and N. Read, "Nonabelions in the fractional quantum hall effect," Nuclear Physics B 360, 362 - 396 (1991).

[9] B. Blok and X. Wen, "Many-body systems with non-abelian statistics," Nuclear Physics B 374, 615 - 646 (1992).

[10] N. Read and E. Rezayi, "Beyond paired quantum hall states: Parafermions and incompressible states in the first excited landau level," Phys. Rev. B 59, 8084-8092 (1999).

[11] E. Ardonne, R. Kedem, and M. Stone, "Filling the Bose sea: symmetric quantum Hall edge states and affine characters," Journal of Physics A: Mathematical and General 38, 617-636 (2004). 
[12] N. Read and D. Green, "Paired states of fermions in two dimensions with breaking of parity and time-reversal symmetries and the fractional quantum hall effect," Phys. Rev. B 61, 10267-10297 (2000).

[13] E. H. Fradkin, C. Nayak, A. Tsvelik, and F. Wilczek, "A Chern-Simons effective field theory for the Pfaffian quantum Hall state," Nucl. Phys. B516, 704-718 (1998), arXiv:cond-mat/9711087 [cond-mat] .

[14] E. H. Fradkin, C. Nayak, and K. Schoutens, "Landau-Ginzburg theories for nonAbelian quantum Hall states," Nucl. Phys. B546, 711-730 (1999), arXiv:cond-mat/9811005 [cond-mat] .

[15] H. Goldman, R. Sohal, and E. Fradkin, "Landau-ginzburg theories of non-abelian quantum hall states from non-abelian bosonization," Phys. Rev. B 100, 115111 (2019).

[16] O. Aharony, "Baryons, monopoles and dualities in Chern-Simons-matter theories," J. High Energy Phys. 02, 93 (2016).

[17] O. Aharony, G. Gur-Ari, and R. Yacoby, " $\mathrm{d}=3$ bosonic vector models coupled to Chern-Simons gauge theories," J. High Energy Phys. 03, 37 (2012).

[18] S. Giombi, S. Minwalla, S. Prakash, S. P. Trivedi, S. R. Wadia, and X. Yin, "ChernSimons theory with vector fermion matter," Eur. Phys. J. C 72, 2112 (2012).

[19] S. G. Naculich and H. J. Schnitzer, "Duality between $S U(N)_{k}$ and $S U(k)_{N}$ WZW models," Nuclear Physics B 347, 687 - 742 (1990).

[20] S. Naculich, H. Riggs, and H. Schnitzer, "Group-level duality in WZW models and Chern-Simons theory," Physics Letters B 246, 417 - 422 (1990).

[21] M. Camperi, F. Levstein, and G. Zemba, "The large N limit of Chern-Simons gauge theory," Physics Letters B 247, 549 - 554 (1990).

[22] P.-S. Hsin and N. Seiberg, "Level/rank Duality and Chern-Simons-Matter Theories," JHEP 09, 095 (2016), arXiv:1607.07457 [hep-th] .

[23] E. Ardonne and K. Schoutens, "New class of non-abelian spin-singlet quantum hall states," Phys. Rev. Lett. 82, 5096-5099 (1999).

[24] E. Ardonne, N. Read, E. Rezayi, and K. Schoutens, "Non-abelian spin-singlet quantum hall states: wave functions and quasihole state counting," Nuclear Physics B 607, 549 - 576 (2001).

[25] Y. Fuji and P. Lecheminant, "Non-abelian $s u(n-1)$-singlet fractional quantum hall states from coupled wires," Phys. Rev. B 95, 125130 (2017).

[26] N. Seiberg, T. Senthil, C. Wang, and E. Witten, "A Duality Web in 2+1 Dimensions and Condensed Matter Physics," Annals of Physics 374, 395 - 433 (2016).

[27] A. Karch and D. Tong, "Particle-Vortex Duality from 3D Bosonization," Phys. Rev. X 


\section{6, 031043 (2016).}

[28] D. F. Mross, J. Alicea, and O. I. Motrunich, "Symmetry and duality in bosonization of two-dimensional Dirac fermions," Phys. Rev. X 7, 041016 (2017).

[29] H. Goldman and E. Fradkin, "Loop Models, Modular Invariance, and Three Dimensional Bosonization," Phys. Rev. B 97, 195112 (2018), arXiv:1801.04936 [cond-mat.str-el] .

[30] X.-G. Wen, "Projective construction of non-abelian quantum hall liquids," Phys. Rev. B 60, 8827-8838 (1999).

[31] M. Barkeshli and X.-G. Wen, "Effective field theory and projective construction for $Z_{k}$ parafermion fractional quantum hall states," Phys. Rev. B 81, 155302 (2010).

[32] M. Barkeshli and X.-G. Wen, "Anyon condensation and continuous topological phase transitions in non-abelian fractional quantum hall states," Phys. Rev. Lett. 105, 216804 (2010).

[33] D. Radicevic, D. Tong, and C. Turner, "Non-Abelian 3d Bosonization and Quantum Hall States," JHEP 12, 067 (2016), arXiv:1608.04732 [hep-th] .

[34] C. Nayak, S. H. Simon, A. Stern, M. Freedman, and S. Das Sarma, "Non-abelian anyons and topological quantum computation," Rev. Mod. Phys. 80, 1083-1159 (2008).

[35] S.-S. Lee, S. Ryu, C. Nayak, and M. P. A. Fisher, "Particle-hole symmetry and the $\nu=\frac{5}{2}$ quantum hall state," Phys. Rev. Lett. 99, 236807 (2007).

[36] M. Levin, B. I. Halperin, and B. Rosenow, "Particle-hole symmetry and the pfaffian state," Phys. Rev. Lett. 99, 236806 (2007).

[37] Z. Komargodski and N. Seiberg, "A symmetry breaking scenario for $\mathrm{QCD}_{3}$," JHEP 01 , 109 (2018), arXiv:1706.08755 [hep-th] .

[38] R. Argurio, M. Bertolini, F. Mignosa, and P. Niro, "Charting the phase diagram of $\mathrm{QCD}_{3}$," JHEP 08, 153 (2019), arXiv:1905.01460 [hep-th] .

[39] A. Baumgartner, "Phases of flavor broken QCD 3 ," JHEP 10, 288 (2019), arXiv:1905.04267 [hep-th] .

[40] A. Armoni, T. T. Dumitrescu, G. Festuccia, and Z. Komargodski, "Metastable vacua in large-N QCD 3 ," JHEP 01, 004 (2020), arXiv:1905.01797 [hep-th] .

[41] A. Baumgartner, "Flavor broken $\mathrm{QCD}_{3}$ at large N," Journal of High Energy Physics 2020, 145 (2020), arXiv:2005.11339 [hep-th] .

[42] M. E. Peskin, "Mandelstam-'t Hooft duality in abelian lattice models," Annals of Physics 113, 122-152 (1978).

[43] C. Dasgupta and B. I. Halperin, "Phase Transition in a Lattice Model of Superconductivity," Phys. Rev. Lett. 47, 1556-1560 (1981).

[44] N. Read, "Lowest-landau-level theory of the quantum hall effect: The fermi-liquid-like 
state of bosons at filling factor one," Phys. Rev. B 58, 16262-16290 (1998).

[45] C. Wang and T. Senthil, "Composite fermi liquids in the lowest landau level," Phys. Rev. B 94, 245107 (2016).

[46] N. R. Cooper, N. K. Wilkin, and J. M. F. Gunn, "Quantum phases of vortices in rotating bose-einstein condensates," Phys. Rev. Lett. 87, 120405 (2001).

[47] Z. Dong and T. Senthil, "Noncommutative field theory and composite fermi liquids in some quantum hall systems," Phys. Rev. B 102, 205126 (2020).

[48] R. Ma and Y.-C. He, "Emergent QCD $_{3}$ Quantum Phase Transitions of Fractional Chern Insulators," (2020), arXiv:2003.05954 [cond-mat.str-el] .

[49] A. Hui, E.-A. Kim, and M. Mulligan, "Non-abelian bosonization and modular transformation approach to superuniversality," Phys. Rev. B 99, 125135 (2019).

[50] C. Cordova, P.-S. Hsin, and K. Ohmori, "Exceptional Chern-Simons-Matter Dualities," SciPost Phys. 7, 56 (2019).

[51] O. Aharony, F. Benini, P.-S. Hsin, and N. Seiberg, "Chern-Simons-matter dualities with SO and USp gauge groups," Journal of High Energy Physics 2017, 72 (2017), arXiv:1611.07874 [cond-mat.str-el] .

[52] D. T. Son, "Is the composite fermion a dirac particle?" Phys. Rev. X 5, 031027 (2015).

[53] N. Seiberg and E. Witten, "Gapped Boundary Phases of Topological Insulators via Weak Coupling," PTEP 2016, 12C101 (2016), arXiv:1602.04251 [cond-mat.str-el] .

[54] R. Jackiw and P. Rossi, "Zero modes of the vortex-fermion system," Nuclear Physics B 190, $681-691$ (1981).

[55] I. Halder and S. Minwalla, "Matter Chern Simons theories in a background magnetic field," Journal of High Energy Physics 2019, 89 (2019), arXiv:1904.07885 [hep-th] .

[56] B. Yang, Y.-H. Wu, and Z. Papić, "Effective abelian theory from a non-abelian topological order in the $\nu=2 / 5$ fractional quantum hall effect," Phys. Rev. B 100, 245303 (2019).

[57] B. Andrews, M. Mohan, and T. Neupert, "Abelian topological order of $\nu=2 / 5$ and 3/7 fractional quantum Hall states in lattice models," (2020), arXiv:2007.08870 [condmat.str-el] .

[58] C. Cordova, P.-S. Hsin, and N. Seiberg, "Global Symmetries, Counterterms, and Duality in Chern-Simons Matter Theories with Orthogonal Gauge Groups," SciPost Phys. 4, 21 (2018).

[59] W. Chen, G. W. Semenoff, and Y.-S. Wu, "Two loop analysis of nonAbelian ChernSimons theory," Phys. Rev. D46, 5521-5539 (1992), arXiv:hep-th/9209005 [hep-th] .

[60] E. Witten, "SL(2,Z) Action On Three-Dimensional Conformal Field Theories With 
Abelian Symmetry," (2003), published in From Fields to Strings vol. 2, p. 1173, M. Shifman et al, eds., World Scientific, 2005, arXiv:0307041 .

[61] D. Gaiotto, A. Kapustin, N. Seiberg, and B. Willett, "Generalized global symmetries," Journal of High Energy Physics 2015, 172 (2015). 